\title{
Existence and characterization of the values of two player differential games with state constraints
}

\author{
Piernicola Bettiol; Marc Quincampoix ${ }^{\dagger}$ and Richard B. Vinter ${ }^{\ddagger}$
}

June 24, 2019

\begin{abstract}
We consider a two player, zero sum differential game with a cost of Bolza type, subject to a state constraint. It is shown that, under a suitable hypothesis concerning existence of inward pointing velocity vectors for the minimizing player at the boundary of the constraint set, the lower value of the game is Lipschitz continuous and is the unique viscosity solution (appropriately defined) of the lower Hamilton Jacobi equation. If the inward pointing hypothesis is satisfied by the maximizing player's velocity set, then the upper game is Lipschitz continuous and is the unique solution of the upper Hamilton Jacobi equation. Under the classical Isaacs condition, the upper and lower Hamilton Jacobi equations coincide. In this case, even if the inward pointing hypothesis is satisfied w.r.t. both players, the value of the game might fail to exist; however imposing stronger constraint qualifications (involving the existence of inward pointing vectors associated with saddle points for the Hamiltonian) the game value does exist and is the unique solution to this Hamilton Jacobi equation. The novelty of our work resides in the fact that we permit the two players' controls to be completely coupled within the dynamic constraint, state constraint functional and the cost, in contrast to earlier work, in which the players' controls are decoupled w.r.t. the dynamics and state constraint, and interaction between them only occurs through the cost function. Furthermore, the inward pointing hypotheses that we impose are of a verifiable nature and less restrictive than those earlier employed.
\end{abstract}

Keywords: Differential Games, State Constraints.

\section{Introduction}

We shall consider a two-player differential game with state-constraints. The dynamic constraint for the game, which relate the state trajectory $x($.$) to the control actions u($.$) and v($.$) of each$ of the two players, takes the form:

$$
\left\{\begin{array}{l}
\dot{x}(t)=f(x(t), u(t), v(t)), \quad \text { for a.e. } t \in\left[t_{0}, T\right] \\
u(t) \in U \text { for a.e. } t \in\left[t_{0}, T\right] \\
v(t) \in V \text { for a.e. } t \in\left[t_{0}, T\right] \\
x\left(t_{0}\right)=x_{0} \in A \\
x(t) \in A \text { for all } t \in\left[t_{0}, T\right] .
\end{array}\right.
$$

* Laboratoire de Mathématiques, Université de Bretagne Occidentale, 6 Avenue Victor Le Gorgeu, 29200 Brest, France, e-mail: piernicola.bettiol@univ-brest.fr

${ }^{\dagger}$ Laboratoire de Mathématiques, Université de Bretagne Occidentale, 6 Avenue Victor Le Gorgeu, 29200 Brest, France, e-mail: Marc.Quincampoix@univ-brest.fr

${ }^{\ddagger}$ Department of Electrical and Electronic Engineering, Imperial College London, Exhibition Road, London, $S W^{\prime}$ 2BT, UK, e-mail: r.vinter@imperial.ac.uk 
Here, $T>0, t_{0} \in[0, T], f: \mathbb{R}^{n} \times \mathbb{R}^{m_{1}} \times \mathbb{R}^{m_{2}} \rightarrow \mathbb{R}^{n}$ is a given function, $A \subset \mathbb{R}^{n}, U \subset \mathbb{R}^{m_{1}}$ and $V \subset \mathbb{R}^{m_{2}}$ are given closed sets. For any left end-point $x_{0} \in A$, any initial time $t_{0} \in[0, T]$ and any choice of measurable controls $(u(),. v()):.\left[t_{0}, T\right] \rightarrow U \times V$, we denote by $x\left[t_{0}, x_{0} ; u(),. v().\right]($. the solution of system (1) (which always exists and is unique under our assumptions).

For arbitrary initial data $\left(t_{0}, x_{0}\right) \in[0, T] \times A$, we associate with controls $(u($.$) and v()$.$) , chosen$ by each of the two players, the following cost functional:

$$
J\left(t_{0}, x_{0} ; u(.), v(.)\right):=\int_{t_{0}}^{T} L(t, x(t), u(t), v(t)) d t+g(x(T)),
$$

in which $x(t)=x\left[t_{0}, x_{0} ; u(),. v().\right](t)$. The function $L: \mathbb{R} \times \mathbb{R}^{n} \times \mathbb{R}^{m_{1}} \times \mathbb{R}^{m_{2}} \rightarrow \mathbb{R}$ is called Lagrangian (or running cost) and $g: \mathbb{R}^{n} \rightarrow \mathbb{R}$ is the final cost.

The aim of the first player is to choose $u($.$) to minimize the cost function and that of the second$ player to choose $v($.$) to maximize it. These objectives are, of course, in conflict. The precise$ nature of 'control' (non-anticipative or open loop) employed by the players will be made precise below.

We shall assume that the data for system (1) and the cost (2) satisfy the following four hypotheses, which we shall refer to as 'standing hypotheses':

(H1): $U \subset \mathbb{R}^{m_{1}}$ and $V \subset \mathbb{R}^{m_{2}}$ are closed sets; $f(x, . .$.$) is \mathcal{B}^{m_{1}} \times \mathcal{B}^{m_{2}}$ measurable for each $x$; $L(t, x, .,$.$) is \mathcal{B}^{m_{1}} \times \mathcal{B}^{m_{2}}$ measurable for each $(t, x)$;

(here, $\mathcal{B}^{m_{i}}$ is the collection of Borel sets of $\mathbb{R}^{m_{i}}$ )

(H2): there exists $M>0$ such that

$|f(x, u, v)| \leq M(1+|x|)$ and $|L(t, x, u, v)| \leq M(1+|x|)$, for all $(t, x) \in[0, T] \times \mathbb{R}^{n}, u \in$ $U, v \in V$

(H3): for every $R>0$, there exist $k_{f}>0, k_{g}>0$ and $k_{L}>0$ such that

$\left|f(x, u, v)-f\left(x^{\prime}, u, v\right)\right| \leq k_{f}\left|x-x^{\prime}\right|, \quad$ for all $t \in[0, T], x, x^{\prime} \in R \mathbb{B}, u \in U$ and $v \in V$;

$\left|L(t, x, u, v)-L\left(t^{\prime}, x^{\prime}, u, v\right)\right| \leq k_{L}\left(\left|t-t^{\prime}\right|+\left|x-x^{\prime}\right|\right)$,

for all $t, t^{\prime} \in[0, T], x, x^{\prime} \in R \mathbb{B}, u \in U$ and $v \in V$;

$\left|g(x)-g\left(x^{\prime}\right)\right| \leq k_{g}\left|x-x^{\prime}\right|, \quad$ for all $x, x^{\prime} \in R \mathbb{B}$;

(here, $\mathbb{B}$ is the closed unit ball in Euclidean space, and $R \mathbb{B}$ is the closed ball of radius $R$ )

(H4): $A$ is a set with non-empty interior having representation:

$$
A=\left\{x \in \mathbb{R}^{n}: h(x) \leq 0\right\},
$$

for a given function $h():. \mathbb{R}^{n} \rightarrow \mathbb{R}$ of class $C^{1+}$ (i.e. $h$ is a $C^{1}$ function with locally Lipschitz gradient) such that $\nabla h(x) \neq 0$ whenever $h(x)=0$.

We shall also find it necessary, at different stages of our investigations, to invoke one or other of the following hypotheses 
(CQ1): for every $R>0$ there exists $\eta>0$ such that

$$
\sup _{v \in V} \inf _{u \in U} \nabla h(x) \cdot f(x, u, v) \leq-\eta, \quad x \in \partial A \cap R \mathbb{B}
$$

(CQ2): for every $R>0$ there exists $\eta>0$ such that

$$
\sup _{u \in U} \inf _{v \in V} \nabla h(x) \cdot f(x, u, v) \leq-\eta, \quad x \in \partial A \cap R \mathbb{B}
$$

We shall refer to (CQ1) and (CQ2) as inward point pointing conditions for player 1 and player 2 , respectively. We shall also invoke a strengthening of the standing hypotheses, namely:

(H5): $U \subset \mathbb{R}^{m_{1}}$ and $V \subset \mathbb{R}^{m_{2}}$ are compact sets; $f(x, . .$.$) is continuous for each x ; L(t, x, . .$.$) is$ continuous for each $(t, x)$.

Given a subinterval $\left[t_{0}, T_{0}\right] \subset[0, T]$ we shall write

$$
\begin{aligned}
\mathcal{U}\left[t_{0}, T_{0}\right]:=\left\{u(.):\left[t_{0}, T_{0}\right] \rightarrow U \text { measurable }\right\} \\
\mathcal{V}\left[t_{0}, T_{0}\right]:=\left\{v(.):\left[t_{0}, T_{0}\right] \rightarrow V \text { measurable }\right\}
\end{aligned}
$$

If, in addition we are given a point $x_{0} \in A$, we shall consider the sets of admissible (also referred to as feasible) controls, for the left end-point $x_{0}$ on the subinterval $\left[t_{0}, T_{0}\right] \subset[0, T]$, which are defined as follows:

$$
\begin{gathered}
\mathcal{A D}\left(\left[t_{0}, T_{0}\right], x_{0}\right):=\left\{(u(.), v(.)) \in \mathcal{U}\left[t_{0}, T_{0}\right] \times \mathcal{V}\left[t_{0}, T_{0}\right]: x\left[t_{0}, x_{0} ; u(.), v(.)\right](t) \in A, \forall t \in\left[t_{0}, T_{0}\right]\right\} ; \\
\mathcal{U}\left(\left[t_{0}, T_{0}\right], x_{0}\right):=\left\{u(.) \in \mathcal{U}\left[t_{0}, T_{0}\right]: \exists v(.) \text { s.t. }(u(.), v(.)) \in \mathcal{A D}\left(\left[t_{0}, T_{0}\right], x_{0}\right)\right\} ; \\
\mathcal{V}\left(\left[t_{0}, T_{0}\right], x_{0}\right):=\left\{v(.) \in \mathcal{V}\left[t_{0}, T_{0}\right]: \exists u(.) \text { s.t. }(u(.), v(.)) \in \mathcal{A D}\left(\left[t_{0}, T_{0}\right], x_{0}\right)\right\}
\end{gathered}
$$

We shall often consider the case in which $T_{0}=T$. In this case we will employ a simplified notation in which $T_{0}=T$ is not explicitly written:

$$
\mathcal{U}\left(t_{0}, x_{0}\right):=\mathcal{U}\left(\left[t_{0}, T\right], x_{0}\right) \quad \text { and } \quad \mathcal{V}\left(t_{0}, x_{0}\right):=\mathcal{V}\left(\left[t_{0}, T\right], x_{0}\right)
$$

We shall see that, under assumptions (H1)-(H4), if in addition (CQ1) (respectively (CQ2)) is satisfied, then, for all $x_{0} \in A$ and $t_{0} \in[0, T]$, we have (cf. Prop. 3.4 below):

$$
\mathcal{V}\left(t_{0}, x_{0}\right)=\mathcal{V}\left[t_{0}, T\right] \quad \text { and } \quad \mathcal{U}\left(\left[t_{0}, T\right], x_{0}\right) \neq \emptyset
$$

(respectively

$$
\left.\mathcal{U}\left(t_{0}, x_{0}\right)=\mathcal{U}\left[t_{0}, T\right] \text { and } \mathcal{V}\left(\left[t_{0}, T\right], x_{0}\right) \neq \emptyset\right) .
$$

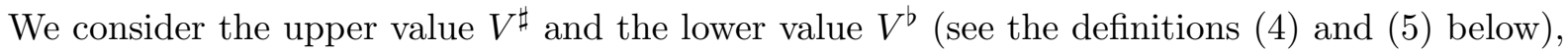
whose definition involves the notion of nonanticipative strategies (in the Varayia-Roxin-ElliotKalton sense). More precisely, fix an initial datum $\left(t_{0}, x_{0}\right) \in\left[t_{0}, T\right] \times A$. We recall that a map $\alpha: \mathcal{V}\left(t_{0}, x_{0}\right) \rightarrow \mathcal{U}\left(t_{0}, x_{0}\right)$ is a non-anticipative strategy for the first player at the point $x_{0}$ if, for any $\tau \in\left[0, T-t_{0}\right]$, for all controls $v_{1}($.$) and v_{2}($.$) belonging to \mathcal{V}\left(t_{0}, x_{0}\right)$, which coincide a.e. on $\left[t_{0}, t_{0}+\tau\right], \alpha\left(v_{1}\right)($.$) and \alpha\left(v_{2}\right)($.$) coincide a.e. on \left[t_{0}, t_{0}+\tau\right]$. Analogously we can define non-anticipative strategies $\beta$ for the second player. Taking the time interval of reference 
$\left[t_{0}, T_{0}\right] \subset[0, T]$, we shall use the following notation for the admissible strategies of, respectively, player 1 and player 2

$$
\begin{gathered}
S_{U}\left(\left[t_{0}, T_{0}\right], x_{0}\right):=\left\{\alpha: \mathcal{V}\left(\left[t_{0}, T_{0}\right], x_{0}\right) \rightarrow \mathcal{U}\left(\left[t_{0}, T_{0}\right], x_{0}\right) \text { s.t. } \alpha(.)\right. \text { is nonanticipative } \\
\text { and } \left.x\left[t_{0}, x_{0} ; \alpha(v), v\right](t) \in A \text { for all } t \in\left[t_{0}, T_{0}\right] \text { and for all } v \in \mathcal{V}\left(\left[t_{0}, T_{0}\right], x_{0}\right)\right\}
\end{gathered}
$$

and

$$
\begin{aligned}
& S_{V}\left(\left[t_{0}, T_{0}\right], x_{0}\right):=\left\{\beta: \mathcal{U}\left(\left[t_{0}, T_{0}\right], x_{0}\right) \rightarrow \mathcal{V}\left(\left[t_{0}, T_{0}\right], x_{0}\right) \text { s.t. } \beta(.)\right. \text { is nonanticipative } \\
& \left.\quad \text { and } x\left[t_{0}, x_{0} ; u, \beta(u)\right](t) \in A \text { for all } t \in\left[t_{0}, T_{0}\right] \text { and for all } u \in \mathcal{U}\left(\left[t_{0}, T_{0}\right], x_{0}\right)\right\} .
\end{aligned}
$$

When $T_{0}=T$, to simplify notation we write $S_{U}\left(t_{0}, x_{0}\right)$ and $S_{V}\left(t_{0}, x_{0}\right)$ the sets of admissible nonanticipative strategies for the first and second player, respectively.

The lower value $V^{b}$ is then defined by:

$$
V^{b}\left(t_{0}, x_{0}\right):=\inf _{\alpha \in S_{U}\left(t_{0}, x_{0}\right)} \sup _{v(.) \in \mathcal{V}\left(t_{0}, x_{0}\right)} J\left(t_{0}, x_{0} ; \alpha(v)(.), v(.)\right)
$$

The upper value function is written as follows:

$$
V^{\sharp}\left(t_{0}, x_{0}\right):=\sup _{\beta \in S_{V}\left(t_{0}, x_{0}\right)} \inf _{u(.) \in \mathcal{U}\left(t_{0}, x_{0}\right)} J\left(t_{0}, x_{0} ; u(.), \beta(u)(.)\right) .
$$

Our goal in this paper is to prove, under hypotheses (H1)-(H5) (referred to as the 'standing assumptions') and also hypothesis (CQ1), that the lower value function is a Lipschitz continuous function that is characterized as the unique uniformly continuous viscosity solution of an appropriate Hamilton-Jacobi-Isaacs (HJI) equation. Analogous properties of the upper value function are established, when (CQ2) replaces (CQ1). We establish also that, under the standing hypotheses, when the Isaacs condition and both (CQ1) and (CQ2), strengthened to include (CQ3) (or (CQ4)) below, are satisfied, the value functions coincide, i.e. the game has a value.

The zero sum differential games literature has, since its inception [15], heavily focused on conditions under which the game has a value. But the lower game is of independent importance, because of its relevance to robust controller design for bounded disturbances. This is a wellestablished field of control engineering design which, it is argued, is better suited to applications involving low frequency, persistent disturbances than those based on stochastic models. One well-developed approach for 'bounded disturbances' robust design, based on 'worst case disturbance' analysis of the effects of the disturbance, involves solving a lower game, in which the controller and disturbance in the controller design problem are interpreted as the first and second control in the lower game, respectively; the lower cost function provides an assessment of the quality of a chosen robust controller design strategy, regarding disturbance suppression and other objectives [4]. By including a state constraint set in the formulation of the lower game, we can take account of the presence of a 'safe region' of the state space. Here, the requirement that the chosen feedback control maintains the state in the safe region, regardless of the disturbance, is built into the design specifications.

A link between the lower value function and viscosity solutions to a HJI equation, with potential application to robust control design, is established in this paper, only when the constraint qualification (CQ1) is satisfied. (CQ1) requires that there exist control values driving the state into the interior of the state constrained region, that the disturbances are 'matched' to the control action, and that the control actuator is designed to have sufficient power to dominate the anticipated bounded disturbances. Robust control design methodologies based on such assumptions 
are standard in the robust control literature, see, e.g. [13].

Aspects of the state-constrained zero sum differential games in this paper that deal with the existence of a value are less relevant to practical control engineering. This is because existence of a value is established only under the assumption that (CQ1), (CQ2) and (CQ3) (or (CQ4)) are satisfied. This is a highly restrictive hypothesis, since (CQ1) and (CQ2), which require that, simultaneously, the control dominates the disturbance and the disturbance dominates the control, are in some sense in conflict. Fortunately, as we have argued, it is only the lower game that is relevant to robust controller design.

Our treatment of state-constrained zero-sum differential games differs from earlier work by Koike [16] in this subject, in three respects. First, we replace the implicit uniform controllability hypotheses in Koike by directly verifiable constraint qualifications (CQ1) or (CQ2). Second, we show that the lower and upper associated value functions are unique viscosity solutions of HJI equations for the upper and lower games respectively, in a simple sense that avoids redefinition of the Hamiltonians on the boundary of the state constraint set, as well consideration of lower and upper envelope solutions, employed in [16]. Third, we provide conditions under which the game has a value.

The paper is organized as follows. We state our main results in Section 2. Section 3 is devoted to nonanticipative constructions of feasible controls and strategies. In Section 4 we establish the Lipschitz regularity of the upper and lower values, characterizing them as constrained viscosity solutions of an Hamilton-Jacobi-Isaacs equation. We provide a comparison result in Section 5.

\section{Main results}

In this section we state our main results, which characterize the lower value, the upper value, and the value functions as generalized solutions in the viscosity sense to the following HJI equation:

$$
\left\{\begin{aligned}
-\partial_{t} W(t, x)+H\left(t, x, \partial_{x} W(t, x)\right) & =0 & \text { on }[0, T) \times A \\
W(T, x) & =g(x) & \text { on } A,
\end{aligned}\right.
$$

in which the function $H: \mathbb{R} \times \mathbb{R}^{n} \times \mathbb{R}^{n} \rightarrow \mathbb{R}$ is the Hamiltonian associated with either the lower or upper game.

Take the (un-max-minimized) Hamiltonian function for the differential game associated with (1)-(2) to be:

$$
\mathcal{H}(t, x, p, u, v):=-f(x, u, v) \cdot p-L(t, x, u, v) .
$$

The lower Hamiltonian and the upper Hamiltonian are defined respectively to be

$$
H^{\mathrm{b}}(t, x, p):=\inf _{v \in V} \sup _{u \in U} \mathcal{H}(t, x, p, u, v)
$$

and

$$
H^{\sharp}(t, x, p):=\sup _{u \in U} \inf _{v \in V} \mathcal{H}(t, x, p, u, v) .
$$

Clearly we have $H^{\sharp} \leq H^{b}$. 
We adopt the following definition of viscosity supersolution and subsolution for state constrained problems, namely.

Definition 2.1 (i): [Viscosity supersolution] $A$ continuous function $W:[0, T] \times A \longrightarrow \mathbb{R}$ is a viscosity supersolution on $D \subset[0, T) \times A$ of the Hamilton-Jacobi-Isaacs equation (6) if for any test function $\varphi: \mathbb{R} \times \mathbb{R}^{n} \rightarrow \mathbb{R}$ of class $\mathcal{C}^{1}$ such that $W-\varphi$ has a local minimum (relative to $[0, T] \times A$ ) at $\left(t_{0}, x_{0}\right) \in D$, then

$$
-\partial_{t} \varphi\left(t_{0}, x_{0}\right)+H\left(t_{0}, x_{0}, \partial_{x} \varphi\left(t_{0}, x_{0}\right)\right) \geq 0 .
$$

(ii): [Viscosity subsolution] A continuous function $W:[0, T] \times A \longrightarrow \mathbb{R}$ is a viscosity subsolution on $D \subset[0, T) \times A$ of (6) if for all $\varphi: \mathbb{R} \times \mathbb{R}^{n} \rightarrow \mathbb{R}$ of class $\mathcal{C}^{1}$ such that $W-\varphi$ has a local maximum (relative to $[0, T] \times A)$ at $\left(t_{0}, x_{0}\right) \in D$, then

$$
-\partial_{t} \varphi\left(t_{0}, x_{0}\right)+H\left(t_{0}, x_{0}, \partial_{x} \varphi\left(t_{0}, x_{0}\right)\right) \leq 0 .
$$

We say that a continuous function $W$ is a viscosity solution on $D \subset[0, T) \times A$ of (6) if it is simultaneously a supersolution and a subsolution of (6) on $D \subset[0, T) \times A$.

Theorem 2.2 Assume that conditions (H1)-(H4) are satisfied.

(i): Suppose, in addition, that (CQ1) is verified. Then, the lower value function $V^{b}$ is locally Lipschitz continuous.

(ii): Suppose, in addition, that (CQ1) and (H5) are verified. Then $V^{b}$ is a viscosity supersolution on $[0, T) \times A$ and a viscosity subsolution on $[0, T) \times$ int $A$ of equation (6) in which $H=H^{\mathrm{b}}$

(iii): Suppose, in addition, that (CQ1) and (H5) are verified, and $A$ is compact. Then $V^{b}$ is the unique function which is simultaneously a supersolution on $[0, T) \times A$ and a subsolution on $[0, T) \times \operatorname{int} A$ of $(6)$, with $H=H^{b}$, in the class of continuous functions.

Observe that $V^{b}$ is a constrained viscosity solution of (6) in the sense of [21] (cf. [2]).

Theorem 2.3 Assume that (H1)-(H4) are satisfied.

(i): Suppose, in addition, that (CQ2) is verified. Then, the lower value function $V^{\sharp}$ is locally Lipschitz continuous.

(ii): Suppose, in addition, that (CQ2) and (H5) are verified. Then $V^{\sharp}$ is a viscosity supersolution on $[0, T) \times \operatorname{int} A$ and a viscosity subsolution on $[0, T) \times A$ of equation (6) in which $H=H^{\sharp}$.

(iii): Suppose, in addition, that (CQ2) and (H5) are verified, and $A$ is compact. Then $V^{\sharp}$ is the unique function which is simultaneously a viscosity supersolution on $[0, T) \times$ int $A$ and a viscosity subsolution on $[0, T) \times A$ of equation (6), with $H=H^{\sharp}$, in the class of continuous functions.

The proofs of these theorems, which are given in later sections of the paper, are built up in several stages. To be more precise, properties (i) and (ii) of Thms 2.2 and 2.3 follow respectively from Prop. 4.2 and Thm. 4.3 below. The comparison Thm. 5.1 establishes part (iii) of Thm. 2.2 and Thm. 2.3. 
We highlight the fact that, for the proof of the Lipschitz regularity of the two value functions, assumption (H5) is not required. It is invoked only when we seek to interpret the values as viscosity subsolutions or supersolutions of (6).

We introduce additional constraint qualifications to derive further properties of the game values.

(CQ3): For every $x_{0} \in \partial A$ and for every $p_{0} \in \mathbb{R}^{n}$ there exist $u_{0} \in U$ and $v_{0} \in V$ such that

(i) $\mathcal{H}\left(t_{0}, x_{0}, p_{0}, u_{0}, v_{0}\right)=\inf _{v \in V} \sup _{u \in U} \mathcal{H}\left(t_{0}, x_{0}, p_{0}, u, v\right)$ and

(ii) $\nabla h\left(x_{0}\right) \cdot f\left(x_{0}, u_{0}, v_{0}\right)<0$.

(CQ4): For every $x_{0} \in \partial A$ and for every $p_{0} \in \mathbb{R}^{n}$ there exist $u_{0} \in U$ and $v_{0} \in V$ such that

(i) $\mathcal{H}\left(t_{0}, x_{0}, p_{0}, u_{0}, v_{0}\right)=\sup _{u \in U} \inf _{v \in V} \mathcal{H}\left(t_{0}, x_{0}, p_{0}, u, v\right)$ and

(ii) $\nabla h\left(x_{0}\right) \cdot f\left(x_{0}, u_{0}, v_{0}\right)<0$.

Observe that the two conditions (CQ3) and (CQ4) coincide when the Isaacs condition, namely

$$
H^{b}(t, x, p)=H^{\sharp}(t, x, p), \quad \forall(t, x, p) \in[0, T) \times A \times \mathbb{R}^{n},
$$

is satisfied.

Theorem 2.4 Assume that (H1)-(H5), (CQ1) and (CQ2) hold true. Suppose moreover that the Isaacs condition (9) and (CQ3) (or equivalently (CQ4)) are satisfied. Then, the game has a value namely: $V^{\sharp}=V^{b}$ and $V:=V^{\sharp}=V^{b}$ is a viscosity solution on $[0, T) \times A$ of (6) with $H=H^{b}=H^{\sharp}$. If, in addition, $A$ is compact, then $V$ is the unique the unique continuous viscosity solution on $[0, T) \times A$ of (6) with $H=H^{b}=H^{\sharp}$.

This theorem is a direct consequence of Cor. 4.6 and Thm. 5.1.

Remarks 2.5 (i) The assertions of Thms 2.2 and 2.3 remain valid if we replace the Hamiltonians $H^{b}$ and $H^{\sharp}$ with the Hamiltonians $H_{-}$and $H_{+}$, respectively, where:

$H_{-}(t, x, p):=\inf _{v \in V} \sup _{u \in U(x, v)} \mathcal{H}(t, x, p, u, v), \quad$ and $\quad H_{+}(t, x, p):=\sup _{u \in U} \inf _{v \in V(x, u)} \mathcal{H}(t, x, p, u, v)$.

Here, $U(x, v):=\left\{u \in U \mid f(x, u, v) \in T_{A}(x)\right\}$ and $V(x, u):=\left\{v \in V \mid f(x, u, v) \in T_{A}(x)\right\}$, in which $T_{A}(x)$ denotes the Clarke tangent cone to the set $A$ at $x$. Notice that $H_{-}$and $H_{+}$ are in general discontinuous, therefore, in this case, the notion of viscosity solution requires consideration of the (upper and lower) semicontinuous envelopes of the Hamiltonians $H_{-}$ and $H_{+}$, indicated by the upper and lower '*' notation (cf. [16, 2]). But using the fact that, under our hypotheses, we have

$$
H_{-}(t, x, p)=H^{b}(t, x, p), \quad H_{+}(t, x, p)=H^{\sharp}(t, x, p) \quad \text { for all } x \in \operatorname{int} A,
$$

and

$$
H_{-}^{*}(t, x, p)=H^{b}(t, x, p), \quad H_{+*}(t, x, p)=H^{\sharp}(t, x, p) \quad \text { for all } x \in \partial A,
$$

we can easily reduce the analysis to the case when we consider the reference lower and upper Hamiltonians $H^{b}$ and $H^{\sharp}$. We also observe that, if all the assumptions of Thm. 2.4 are satisfied (included the Isaacs condition (9), precisely with $H^{b}$ and $H^{\sharp}$ ), then the value $V:=V^{\sharp}=V^{b}$ is still the unique bounded uniformly continuous viscosity solution on $D \subset[0, T) \times A$ of (6) with $H=H_{-}$and, also, with $H=H_{+}$.

(ii) Similar results were earlier proved in [5], but only in the case of separated dynamics. 
(iii) Observe that, if the standing hypotheses are merely supplemented by the Isaacs condition and (CQ1)-(CQ2), from Cor. 4.5 and Thm. 5.1 we can only conclude that $V^{\sharp} \leq V^{b}$ on $[0, T] \times A$. To obtain the existence of the value for the game we need to impose also (CQ3) (or (CQ4)). While (CQ3) and (CQ4) are very strong, the value of the game may not exist, if they are not imposed. This is illustrated by Example 1 below.

Example 1. Consider the following two-player differential game with state-constraints:

$$
\left\{\begin{array}{l}
\dot{x}(t)=u(t)+v(t), \quad \text { for a.e. } t \in\left[t_{0}, 1\right] \\
u(t) \in U:=[-3,+1] \quad \text { for a.e. } t \in\left[t_{0}, 1\right] \\
v(t) \in V:=[-2,+2] \quad \text { for a.e. } t \in\left[t_{0}, 1\right] \\
x\left(t_{0}\right)=x_{0} \in A \\
x(t) \in A:=\{y \in \mathbb{R} \mid x \leq 0\} \quad \text { for all } t \in\left[t_{0}, T\right] .
\end{array}\right.
$$

For arbitrary initial data $\left(t_{0}, x_{0}\right) \in[0,1] \times A$, and controls $(u($.$) and v()$.$) , we consider the$ following cost functional:

$$
J\left(t_{0}, x_{0} ; u(.), v(.)\right):=\int_{0}^{1}(-u(t)+v(t)) d t .
$$

Observe that, for this example, the Isaacs condition and the two constraint qualifications (CQ1) and (CQ2) are satisfied, but (CQ3) (and (CQ4)) is violated. We claim that we have the strict inequality:

$$
V^{b}(0,0)>V^{\sharp}(0,0)
$$

Proof of the claim. Notice that

$$
\mathcal{U}(0,0)=\left\{w(.) \in L^{\infty}: w(t) \in[-3,+1] \text { a.e. }\right\}, \quad \mathcal{V}(0,0)=\left\{w(.) \in L^{\infty}: w(t) \in[-2,+2] \text { a.e. }\right\} .
$$

Consider the lower game. Take any $\epsilon>0$. Then, there exists a non-anticipative strategy $\alpha \in S_{U}(0,0)$ such that $x[0,0 ; \alpha(v), v](t) \in A$ for all $t \in[0,1]$ and $v(.) \in \mathcal{V}(0,0)$, and

$$
V^{b}(0,0) \geq \sup _{v(.) \in \mathcal{V}(0,0)} \int_{0}^{1}(-\alpha(v)(t)+v(t)) d t-\epsilon .
$$

We also know that, for any $v(.) \in \mathcal{V}(0,0), u()=.\alpha(v)($.$) must satisfy the state constraint and$ hence

$$
\int_{0}^{1} u(t) d t \leq-\int_{0}^{1} v(t) d t
$$

It follows that

$$
\sup _{v(.) \in \mathcal{V}(0,0)} J(0,0 ; \alpha(v)(.), v(.)) \geq \sup _{v(.) \in \mathcal{V}(0,0)}\left(2 \times \int_{0}^{1} v(t) d t\right)=4 .
$$

Since $\epsilon>0$ is arbitrary, we deduce that

$$
V^{b}(0,0) \geq 4
$$

Consider now the upper game. Take any $\epsilon>0$. Then, there exists a non-anticipative strategy $\beta \in S_{V}(0,0)$ such that $x[0,0 ; u, \beta(u)](t) \in A$ for all $t \in[0,1]$ and $u(.) \in \mathcal{U}(0,0)$, and

$$
V^{\sharp}(0,0) \leq \inf _{u(.) \in \mathcal{U}(0,0)} \int_{0}^{1}(-u(t)+\beta(u)(t)) d t+\epsilon .
$$


For any $u(.) \in \mathcal{U}(0,0), v()=.\beta(u)($.$) must satisfy$

$$
\int_{0}^{1} v(t) d t \leq-\int_{0}^{1} u(t) d t
$$

It follows that

$$
\inf _{u(.) \in \mathcal{U}(0,0)} J(0,0 ; u(.), \beta(u)(.)) \leq \inf _{u(.) \in \mathcal{U}(0,0)}\left(-2 \times \int_{0}^{1} u(t) d t\right)=-2 .
$$

Since $\epsilon>0$ is arbitrary, we obtain that

$$
V^{\sharp}(0,0) \leq-2,
$$

confirming the claim.

Remark 2.6 It is easy to see that the implicit uniform controllability hypotheses in [16] (and, in turn, the results in [16]) are not applicable for Example 1. On the other hand, the constraint qualifications (CQ1) and (CQ2) are directly verifiable, and Thms 2.2 and 2.3 above characterize respectively the lower value and the upper value functions. Take a slight modification of Example 1 in which we adopt all the previous data, exception made for the control sets of the two players: now we have $U:=\{-3,+1\}$ and $V:=\{-2,+2\}$. In this case not only (CQ1) and (CQ2) are satisfied but also the implicit uniform controllability hypotheses in [16] hold true, and therefore Thms 2.2, 2.3 and the results in [16] are applicable. Still, the value of the game does not exist: indeed, some routine analysis show that $V^{b}(t, x)=1-t$ if $x \leq 3 t-3$ and $V^{b}(t, x)=x-4 t+4$ if $3 t-3<x \leq 0$, while $V^{\sharp}(t, x)=1-t$ if $x \leq 3 t-3$ and $V^{\sharp}(t, x)=2 t-x-2$ if $3 t-3<$ $x \leq 0$. Morevover, consider for instance the upper value $V^{\sharp}$ : as stated in Thm. 2.3 this is the unique function which is simultaneously a viscosity supersolution on $[0, T) \times \operatorname{int} A$ and a viscosity subsolution on $[0, T) \times A$ of equation (6), with $H=H^{\sharp}$, in the class of continuous functions. But, $V^{\sharp}$ becomes the viscosity solution on $[0, T) \times A$ of equation (6), when we take the Hamiltonian $H=H_{+}$defined in Rmk. 2.5-(i) (which, for this particular case, coincides the 'inner upper Hamiltonian' considered by Koike [16]). So, this modified example shows that, if we want to derive the existence of the game value via a comparison argument, it is better to consider the (standard) upper Hamiltonian $H^{\sharp}$ and lower Hamiltonian $H^{b}$, instead of the 'inner' upper Hamiltonian $H_{+}$and the 'inner' lower Hamiltonian $H_{-}$, even if the 'inner' Hamiltonians $H_{+}$and $H_{-}$might provide a viscosity solution characterization up to the boundary of $A$.

\section{State constrained control systems: nonanticipative construc- tions of feasible controls}

Consider the state-constrained control system, described as follows:

$$
\begin{aligned}
& \dot{x}(t)=\tilde{f}(t, x(t), u(t)) \text { a.e. } t \in[0, T] \\
& u(t) \in \widetilde{U}(t) \\
& x(t) \in A \quad \text { for all } t \in[0, T],
\end{aligned}
$$

in which $\tilde{f}(., .,):. \mathbb{R} \times \mathbb{R}^{n} \times \mathbb{R}^{m} \rightarrow \mathbb{R}^{n}$ is a given function, and $\widetilde{U}():. \mathbb{R} \leadsto \mathbb{R}^{m}$ is a given multifunction.

We shall refer to a couple $(x(),. u()$.$) , comprising a measurable function u():. I \rightarrow \mathbb{R}^{m}$ and an absolutely continuous function $x():. I \rightarrow \mathbb{R}^{n}$ which satisfy $\dot{x}(t)=\tilde{f}(t, x(t), u(t))$ and $u(t) \in \widetilde{U}(t)$ 
a.e., as a process (on the subinterval $I \subset[0, T]$ ). The function $x($.$) is called a state trajectory$ and the function $u($.$) is called a control function. If x($.$) satisfies the state constraint (11), the$ process is 'feasible'.

We shall assume that the control system data satisfy the following hypotheses, in which $r_{0}$ is some positive real number. There exist constants $\rho>0, \eta>0$ and $M>0$, and $\tilde{k}_{f}(.) \in L^{1}([0, T], \mathbb{R})$ such that:

$(\mathrm{H} 1)^{\prime}: \tilde{f}(., x,$.$) is \mathcal{L} \times \mathcal{B}^{m}$ measurable for each $x$, where $\mathcal{L}$ is the collection of Lebesgue measurable sets of $\mathbb{R}$ and $\mathcal{B}^{m}$ the collection of Borel sets of $\mathbb{R}^{m}$; the set-valued map $\widetilde{U}($.$) has Borel-$ measurable graph.

$(\mathrm{H} 2)^{\prime}:|\tilde{f}(t, x, u)| \leq M(1+|x|) \quad$ for all $(t, x) \in[0, T] \times \mathbb{R}^{n}, u \in \widetilde{U}(t)$.

$(\mathrm{H} 3)^{\prime}:\left|\tilde{f}(t, x, u)-\tilde{f}\left(t, x^{\prime}, u\right)\right| \leq \tilde{k}_{f}(t)\left|x-x^{\prime}\right| \quad$ for all $t \in[0, T], x, x^{\prime} \in e^{M T}\left(1+r_{0}\right) \mathbb{B}$ and $u \in U(t)$.

(CQ): (Inward pointing condition)

$$
\inf _{u \in \widetilde{U}(t)} \nabla h(x) \cdot \tilde{f}(t, x, u) \leq-\eta
$$

for all $(t, x) \in[0, T] \times e^{M T}\left(1+r_{0}\right) \mathbb{B}$ for which $-\rho \leq h(x) \leq 0$.

Employing the $L^{\infty}$-metric on the set of trajectories, and the (Ekeland) metric:

$$
d_{I}\left(u_{1}(.), u_{2}(.)\right)=\text { meas }\left\{t \in I: u_{1}(t) \neq u_{2}(t)\right\},
$$

on the set of controls, we derive linear estimates w.r.t. the left-end points of a reference process and its approximating process. Such estimates, often referred as nonanticipative Filippov-type theorems (cf. [6, 7]), ensure that it is possible to construct approximating feasible controls (and trajectories) in a nonanticipative way, and, therefore, build up suitable nonanticipative strategies.

Here, we restrict attention to the case in which the boundary of $A$ is smooth, illustrating how the approach suggested in [8] can be extended to obtain Filippov's type theorems. The basic idea is to modify the control on a suitable measurable set whose measure is bounded above by a number which depends linearly on the distance of two distinct left-end points. Imposing stronger inward pointing conditions (as in [9] and [18]), we can construct controls with similar properties for state constraints sets that are merely assumed to be closed.

Theorem 3.1 [Nonanticipative Filippov's Theorem] Fix $r_{0}>0$. Assume that hypotheses (H1)'$\left(\mathrm{H}_{3}\right)^{\prime}$, ( $\left.\mathrm{H}_{4}\right)$ and $(\mathrm{CQ})$ are satisfied. Then there exists a constant $K>0$ (whose magnitude depends only on the parameter $r_{0}$ and the data of assumptions (H1)'-(H3)', (H4) and (CQ)) with the following property: given any $\left(\tau, y_{1}\right) \in[0, T] \times\left(\left(\left(r_{0}+1\right) e^{M \tau}-1\right) \mathbb{B} \cap A\right)$ and feasible process $\left(x_{1}(),. u_{1}().\right)$ on $[\tau, T]$ such that $x_{1}(\tau)=y_{1}$, for any $y_{2} \in A \cap\left(\left(r_{0}+1\right) e^{M \tau}-1\right) \mathbb{B}$, and any $d>0$ such that $d \geq\left|y_{2}-y_{1}\right|$, there exists a feasible process $\left(x_{2}(),. u_{2}().\right)$ on $[\tau, T]$ with $x_{2}(\tau)=y_{2}$ such that the construction of $u_{2}($.$) is nonanticipative, and$

$$
\begin{aligned}
& x_{2}(t) \in \operatorname{int} A, \text { for all } t \in(\tau, T], \\
& d_{[\tau, T]}\left(u_{1}(.), u_{2}(.)\right) \leq K d, \\
& \left\|x_{1}(.)-x_{2}(.)\right\|_{L^{\infty}(\tau, T)} \leq K d .
\end{aligned}
$$


We start by proving a local version of the theorem in the form of the following lemma.

Lemma 3.2 Fix $r_{0}>0$. Assume that $\left(H_{1}\right)^{\prime}-\left(H_{3}\right)^{\prime},\left(H_{4}\right)$ and $(C Q)$ are satisfied. Then, there exist constants $\delta_{0}>0, K_{0}>0$ and $K_{1}>1$ (depending only on the parameter $r_{0}$ and the data provided by assumptions $\left(\mathrm{H}_{1}\right)^{\prime}-\left(\mathrm{H}_{3}\right)^{\prime},\left(\mathrm{H}_{4}\right)$ and $\left.(\mathrm{CQ})\right)$ satisfying the following property: take any $\tau \in[0, T], y, y^{\prime} \in A \cap\left(\left(r_{0}+1\right) e^{M \tau}-1\right) \mathbb{B}$, any $d>0$ such that $d \geq\left|y-y^{\prime}\right|$, and any feasible process $(x(),. u()$.$) on [\tau, T]$ with $x(\tau)=y$. Then, there exists a process $\left(x^{\prime}(),. u^{\prime}().\right)$ on $[\tau, T]$ such that $x^{\prime}(\tau)=y^{\prime}$,

$$
x^{\prime}(t) \in \operatorname{int} A \quad \text { for all } t \in\left(\tau,\left(\tau+\delta_{0}\right) \wedge T\right],
$$

the construction of $u^{\prime}($.$) is nonanticipative, and$

$$
\begin{aligned}
& d_{[\tau, T]}\left(u(.), u^{\prime}(.)\right) \leq K_{0} d \\
& \left\|x(.)-x^{\prime}(.)\right\|_{L^{\infty}(\tau, T)} \leq K_{1}\left(\left|y-y^{\prime}\right|+d_{[\tau, T]}\left(u(.), u^{\prime}(.)\right)\right) .
\end{aligned}
$$

Proof. Fix any $r_{0}>0, \tau \in[0, T]$, and $y, y^{\prime} \in A \cap\left(\left(r_{0}+1\right) e^{M \tau}-1\right) \mathbb{B}$, and any $d>0$ such that $d \geq\left|y-y^{\prime}\right|$. The number $R_{0}:=e^{M T}\left(1+r_{0}\right)$ is an upper bound on the magnitude of all state trajectories $x($.$) on [\tau, T]$ such that $x(\tau) \in\left(\left(r_{0}+1\right) e^{M \tau}-1\right) \mathbb{B}$, and, from $(\mathrm{H} 2)^{\prime}, M\left(1+R_{0}\right)$ is an upper bound for the corresponding velocities $\dot{x}($.$) . Write k_{h}$ and $k_{h}^{\prime}$ the Lipschitz constants for $h($.$) and \nabla h($.$) respectively, on R_{0} \mathbb{B}$. Denote by $\omega($.$) a modulus of continuity for the function$ $t \rightarrow \int_{0}^{t} \tilde{k}_{f}(s) d s$

Assumptions (H1)'-(H3)', (H4) and (CQ) guarantee also the existence of $\rho_{0}>0$ and $\bar{\delta}>0$ with the following property: for any $\tau \in[0, T]$ and any process $(x(),. u()$.$) on [\tau,(\tau+\bar{\delta}) \wedge T]$ with $x(\tau) \in A \cap\left(\left(r_{0}+1\right) e^{M \tau}-1\right) \mathbb{B}$, one of the following two cases occurs: either

case 1): $h(x(\tau)) \leq-\rho_{0}$; if we have this initial condition, then $x(t) \in A$ for all $t \in[\tau,(\tau+\bar{\delta}) \wedge T]$

or

case 2): $-\rho_{0}<h(x(\tau)) \leq 0$; in this case there exists a control $\bar{u}():.[\tau,(\tau+\bar{\delta}) \wedge T] \rightarrow \mathbb{R}^{m}$ such that $\bar{u}(t) \in \widetilde{U}(t)$ a.e. and

$$
\nabla h(x(t)) \cdot \tilde{f}(t, x(t), \bar{u}(t)) \leq-\eta \quad \text { a.e. }[\tau,(\tau+\bar{\delta}) \wedge T] .
$$

We define the continuous function $\beta():. \mathbb{R}_{+} \rightarrow \mathbb{R}_{+}$as follows:

$$
\beta(\delta):=2 M\left(1+R_{0}\right)\left(k_{h}^{\prime} M\left(1+R_{0}\right) \delta+k_{h} \omega(\delta)\right) e^{\int_{0}^{T} \tilde{k}_{f}(s) d s} .
$$

Since $\beta($.$) is monotone increasing and \beta(0)=0$, we can choose $\delta_{0}:=\delta\left(r_{0}\right) \in(0, \bar{\delta})$ such that $\beta\left(\delta_{0}\right)<\eta$. We also select $K_{0}:=K\left(r_{0}\right)>0$ such that

$$
K_{0}>\frac{k_{h} e^{\int_{0}^{T} \tilde{k}_{f}(s) d s}}{\eta-\beta\left(\delta_{0}\right)} .
$$

Now we fix any $\tau \in[0, T]$ and a feasible process $(x(),. u()$.$) on [\tau, T]$ such that $x(\tau)=y$. Write $\bar{\tau}:=\left(\tau+\delta_{0}\right) \wedge T$ to simplify notation. We shall construct a second process $\left(x^{\prime}(),. u^{\prime}().\right)$ on $[\tau, T]$ such that $x^{\prime}(\tau)=y^{\prime}$,

$$
x^{\prime}(t) \in \operatorname{int} A \quad \forall t \in(\tau, \bar{\tau}]
$$


and

$$
\begin{aligned}
& d_{[\tau, T]}\left(u(.), u^{\prime}(.)\right) \leq K_{0} d \\
& \left\|x(.)-x^{\prime}(.)\right\|_{L^{\infty}(\tau, T)} \leq K_{1}\left(\left|y-y^{\prime}\right|+d_{[\tau, T]}\left(u(.), u^{\prime}(.)\right)\right) .
\end{aligned}
$$

Consider the (non necessarily feasible) process $(\hat{x}(),. u()$.$) with left-end point \hat{x}(\tau)=y^{\prime}$. We shall assume that case 2) occurs for, otherwise, $\left(x^{\prime}(),. u^{\prime}().\right)=(\hat{x}(),. u()$.$) has already the desired$ properties. Notice that from the Gronwall inequality (cf. for instance [22, Lemma 2.4.4]) we obtain:

$$
\| x(.)-\left.\hat{x}(.)\right|_{L^{\infty}(\tau, t)} \leq e^{\int_{0}^{T} \tilde{k}_{f}(s) d s}\left|y-y^{\prime}\right|, \quad \forall t \in[\tau, T] .
$$

For all $t \in[\tau, \bar{\tau}]$ we denote by $\mathcal{A}(t)$ the measurable set

$$
\mathcal{A}(t)=\left\{s \in[\tau, t] \mid \frac{d}{d s} h(\hat{x}(s)) \geq 0\right\}
$$

where $\frac{d}{d s} h(\hat{x}(s))$ is the total derivative of the map $s \rightarrow h(\hat{x}(s))$. Consider now the process $\left(x^{\prime}(),. u^{\prime}().\right)$ on $[\tau, T]$ such that $x^{\prime}(\tau)=y^{\prime}$ and

$$
u^{\prime}(t)=\left\{\begin{array}{l}
\bar{u}(t) \text { if } t \in[\sigma, T], \quad \frac{d}{d t} h(\hat{x}(t)) \geq 0 \text { and } \operatorname{meas}\{\mathcal{A}(t)\}<K_{0} d \\
u(t) \text { otherwise }
\end{array}\right.
$$

where the control $\bar{u}(t) \in \widetilde{U}(t)$ satisfies the following inward pointing property (recall that we are in case 2):

$$
\nabla h(\hat{x}(t)) \cdot \tilde{f}(t, \hat{x}(t), \bar{u}(t)) \leq-\eta \quad \text { a.e. } t \in[\tau, \bar{\tau}] .
$$

Observe that the construction above is nonanticipative, in the sense that if two controls $u_{1}($. and $u_{2}($.$) are such that u_{1}()=.u_{2}($.$) a.e. on some time interval \left[\tau, \tau^{*}\right] \subset[\tau, T]$, then the corresponding controls $u_{1}^{\prime}($.$) and u_{2}^{\prime}($.$) , defined by (19), satisfy the property u_{1}^{\prime}()=.u_{2}^{\prime}($.$) a.e.$ on $\left[\tau, \tau^{*}\right]$.

Set

$$
\bar{\sigma}:=\sup \left\{t \in[\tau, \bar{\tau}]: \operatorname{meas}\{\mathcal{A}(t)\}<K_{0} d\right\}
$$

Notice that

$$
d_{[\tau, T]}\left(u(.), u^{\prime}(.)\right)=d_{[\tau, \bar{\tau}]}\left(u(.), u^{\prime}(.)\right) \leq \operatorname{meas}\{\mathcal{A}(\bar{\sigma})\} \leq K_{0} d,
$$

and, owing to Gronwall's Inequality, we also have

$$
\begin{aligned}
\left\|x^{\prime}(.)-\hat{x}(.)\right\|_{L^{\infty}(\tau, t)} & \leq 2 M\left(1+R_{0}\right) e^{\int_{0}^{T} \tilde{k}_{f}(s) d s} d_{[\tau, \bar{\tau}]}\left(u(.), u^{\prime}(.)\right) \\
& \leq 2 M\left(1+R_{0}\right) e^{\int_{0}^{T} \tilde{k}_{f}(s) d s} \operatorname{meas}\{[\tau, t \wedge \bar{\sigma}] \cap \mathcal{A}(t)\}, \quad \forall t \in[\tau, T] .
\end{aligned}
$$

Then, from (17) and (20), taking $K_{1}:=\left(1+2 M\left(1+R_{0}\right)\right) e^{\int_{0}^{T} \tilde{k}_{f}(s) d s}$, we also derive

$$
\left\|x(.)-x^{\prime}(.)\right\|_{L^{\infty}(\tau, t)} \leq K_{1}\left(\left|y-y^{\prime}\right|+d_{[\tau, T]}\left(u(.), u^{\prime}(.)\right)\right), \quad \forall t \in[\tau, T] .
$$

It remains to prove that the process $\left(x^{\prime}(),. u^{\prime}().\right)$ is feasible on $[\tau, \bar{\tau}]$, and more precisely $x^{\prime}(t) \in$ int $A$, for all $t \in(\tau, \bar{\tau}]$. 
Clearly, for each $t \in[\tau, \bar{\tau}]$, we obtain

$$
\begin{aligned}
h\left(x^{\prime}(t)\right)= & h\left(x^{\prime}(\tau)\right)+\int_{\tau}^{t} \nabla h\left(x^{\prime}(s)\right) \cdot \tilde{f}\left(s, x^{\prime}(s), u^{\prime}(s)\right) d s \\
\leq & h\left(x^{\prime}(\tau)\right)+\int_{\tau}^{t} \nabla h(\hat{x}(s)) \cdot \tilde{f}\left(s, x^{\prime}(s), u^{\prime}(s)\right) d s \\
\quad & \quad+k_{h}^{\prime} M\left(1+R_{0}\right)|t-\tau|\left\|x^{\prime}(.)-\hat{x}(.)\right\|_{L^{\infty}(\tau, t)} \\
\leq \quad & h\left(x^{\prime}(\tau)\right)+\int_{\tau}^{t} \nabla h(\hat{x}(s)) \cdot \tilde{f}\left(s, \hat{x}(s), u^{\prime}(s)\right) d s \\
& \quad+\left(k_{h}^{\prime} M\left(1+R_{0}\right)|t-\tau|+k_{h} \omega(|t-\tau|)\right)\left\|x^{\prime}(.)-\hat{x}(.)\right\|_{L^{\infty}(\tau, t)} .
\end{aligned}
$$

We claim that $h(x(t))<0$ for all $t \in(\tau, \bar{\tau}]$. We consider the following two possible cases:

Case i): $t \in[\tau, \bar{\sigma}]$. We have

$$
\begin{aligned}
& \int_{\tau}^{t} \nabla h(\hat{x}(s)) \cdot \tilde{f}\left(s, \hat{x}(s), u^{\prime}(s)\right) d s \leq \int_{[\tau, t] \backslash \mathcal{A}(t)} \nabla h(\hat{x}(s)) \cdot \tilde{f}(s, \hat{x}(s), u(s)) d s \\
& \quad+\int_{[\tau, t] \cap \mathcal{A}(t)} \nabla h(\hat{x}(s)) \cdot \tilde{f}(s, \hat{x}(s), \bar{u}(t)) d s \\
& \quad<0-\eta \operatorname{meas}\{\mathcal{A}(t)\} .
\end{aligned}
$$

As a consequence from the definition of $\beta\left(\delta_{0}\right)$ and inequalities (20) and (21), it follows that

$$
h\left(x^{\prime}(t)\right)<-\left(\eta-\beta\left(\delta_{0}\right)\right) \text { meas }\{\mathcal{A}(t)\}<0 .
$$

Case ii): $t \in[\bar{\sigma}, \bar{\tau}]$. In this case, since $h(\hat{x}(\tau))=h\left(x^{\prime}(\tau)\right)$ we have that

$$
\begin{aligned}
& \int_{\tau}^{t} \nabla h(\hat{x}(s)) \cdot \tilde{f}\left(s, \hat{x}(s), u^{\prime}(s)\right) d s=\int_{\tau}^{t} \nabla h(\hat{x}(s)) \cdot \tilde{f}(s, \hat{x}(s), u(s)) d s \\
& \quad+\int_{\tau}^{t} \nabla h(\hat{x}(s)) \cdot\left[\tilde{f}\left(s, \hat{x}(s), u^{\prime}(s)\right)-f(s, \hat{x}(s), u(s))\right] d s \\
& \leq h(\hat{x}(t))-h(\hat{x}(\tau))-\eta \operatorname{meas}\{\mathcal{A}(\bar{\sigma})\}=h(\hat{x}(t))-h\left(x^{\prime}(\tau)\right)-\eta K_{0} d .
\end{aligned}
$$

From this estimate, (16) and inequalities (17), (20) and (21), we derive that

$$
\begin{aligned}
h\left(x^{\prime}(t)\right) & \leq k_{h}\|x(.)-\hat{x}(.)\|_{L^{\infty}(\tau, t)}-\left(\eta-\beta\left(\delta_{0}\right)\right) K_{0} d \\
& \leq\left[k_{h} e^{\int_{0}^{T} \omega(s) d s}-\left(\eta-\beta\left(\delta_{0}\right)\right) K_{0}\right] d \\
& <0 .
\end{aligned}
$$

This confirms our claim and the proof of the lemma is now complete.

Proof of Theorem 3.1. Assume that $(\mathrm{H} 1)^{\prime}-(\mathrm{H} 3)^{\prime},(\mathrm{H} 4)$ and $(\mathrm{CQ})$ are satisfied, and fix $r_{0}>0$. Then consider the constants $\delta_{0}>0, K_{0}>0$ and $K_{1}>1$ provided by Lemma 3.2. Choose the integer $N_{0}$ in such a manner that it is the first index value $i$ for which $\tau+\left(i \delta_{0}\right) \geq T$. Observe 
that $N_{0}$ satisfies $N_{0} \leq T / \delta_{0}+1$, and does not depend on the choice of reference time $\tau$, or the choice of the left-end points $y_{1}$ and $y_{2}$, or the process $\left(x_{1}(),. u_{1}().\right)$.

We recursively apply Lemma 3.2 to the reference process $(x(),. u())=.\left(x_{1}(),. u_{1}().\right)$ on $[\tau, T]$, to obtain a finite sequence of processes $\left(x_{i}^{\prime}(),. u_{i}^{\prime}().\right)$ on $[\tau, T], i=1, \ldots, N_{0}$, where the $i$ th process is an extension of the $(i-1)$ th process, which is feasible on $\left[\tau,\left(\tau+i \delta_{0}\right) \wedge T\right]$ (more precisely $x_{i}^{\prime}(t) \in \operatorname{int} A$ for all $\left.\left(\tau,\left(\tau+i \delta_{0}\right) \wedge T\right]\right)$, and the $i$ th control $u_{i}^{\prime}($.$) is constructed in a$ nonanticipative way. We shall finally take $\left(x_{2}(),. u_{2}().\right):=\left(x_{N_{0}}^{\prime}(),. u_{N_{0}}^{\prime}().\right)$. This process, then, is such that $x_{2}(\tau)=y_{2}, x_{2}(t) \in \operatorname{int} A$ for all $t \in(\tau, T]$, and satisfies all the required properties established in the statement of the proposition.

Writing $\tau_{0}=\tau, \tau_{i}=\tau+\left(i \delta_{0}\right), y=x\left(\tau_{0}\right)=y_{1}, y^{\prime}=x_{1}^{\prime}\left(\tau_{0}\right)=y_{2}$, we have, for $i=1, \ldots, N_{0}$

$$
\begin{aligned}
& d_{\left[\tau_{i-1}, T\right]}\left(u(.), u_{i}^{\prime}(.)\right) \leq K_{0} d_{i-1} \\
& \left\|x(.)-x_{i}^{\prime}(.)\right\|_{L^{\infty}\left(\tau_{i-1}, T\right)} \leq K_{1}\left(\left|x\left(\tau_{i-1}\right)-x_{i}^{\prime}\left(\tau_{i-1}\right)\right|+d_{\left[\tau_{i-1}, T\right]}\left(u(.), u_{i}^{\prime}(.)\right)\right),
\end{aligned}
$$

where $d_{i-1}=\left|x\left(\tau_{i-1}\right)-x_{i}^{\prime}\left(\tau_{i-1}\right)\right|$, for $i=1, \ldots, N_{0}$, and $d_{0}=d\left(\geq\left|y-y^{\prime}\right|\right)$. Therefore, for $i=1, \ldots, N_{0}$, we obtain

$$
d_{\left[\tau_{i-1}, T\right]}\left(u(.), u_{i}^{\prime}(.)\right) \leq K_{0} K_{1}^{i-1}\left(1+K_{0}\right)^{i-1} d
$$

and

$$
\left\|x(.)-x_{i}^{\prime}(.)\right\|_{L^{\infty}\left(\tau_{i-1}, T\right)} \leq K_{1}^{i-1}\left(1+K_{0}\right)^{i-1} d
$$

As a consequence we derive, from (23),

$$
\begin{aligned}
d_{[\tau, T]}\left(u(.), u_{N_{0}}^{\prime}(.)\right) & \leq \sum_{i=1}^{N_{0}} d_{\left[\tau_{i-1}, T\right]}\left(u(.), u_{i}^{\prime}(.)\right) \\
& \leq \sum_{i=1}^{N_{0}} K_{0} K_{1}^{i-1}\left(1+K_{0}\right)^{i-1} d \\
& \leq K_{1}^{N_{0}}\left(1+K_{0}\right)^{N_{0}} d
\end{aligned}
$$

and, from (24),

$$
\begin{aligned}
\left\|x(.)-x_{N_{0}}^{\prime}(.)\right\|_{L^{\infty}(\tau, T)} & \leq \max _{i=1, \ldots, N_{0}}\left\|x(.)-x_{i}^{\prime}(.)\right\|_{L^{\infty}\left(\tau_{i-1}, T\right)} \\
& \leq K_{1}^{N_{0}}\left(1+K_{0}\right)^{N_{0}} d .
\end{aligned}
$$

Thus, taking $K:=K_{1}^{N_{0}}\left(1+K_{0}\right)^{N_{0}}$, all the assertions of the Theorem 3.1 are confirmed.

Assume that we are given initial data and a control function (non necessarily feasible). A modification of the proof of Thm. 3.1 provides a nonanticipative construction of a feasible control for the reference initial data which satisfy some specific inward pointing properties, paying the (sometimes) 'affordable price' of neglecting the distance estimates between the employed controls. In particular we obtain that for any initial data, the set of feasible processes is nonempty.

Proposition 3.3 Fix any $r_{0}>0$. Assume that $\left(\mathrm{H}_{1}\right)^{\prime}-\left(\mathrm{H}_{3}\right)^{\prime}$, (H4) and $(\mathrm{CQ})$ are satisfied. Take any initial time $t_{0} \in[0, T]$ and any $x_{0} \in A \cap\left(e^{M t_{0}}\left(r_{0}+1\right)-1\right) \mathbb{B}$. Consider a measurable function $u($.$) defined on \left[t_{0}, T\right]$ with $u(t) \in \widetilde{U}(t)$ a.e.. We can construct a feasible process $(\tilde{x}(),. \tilde{u}()$.$) on$ $\left[t_{0}, T\right]$ such that $\tilde{x}\left(t_{0}\right)=x_{0}, \tilde{x}(t) \in \operatorname{int} A$ for all $t \in\left(t_{0}, T\right]$, and the map $u(.) \rightarrow \gamma_{t_{0}, x_{0}}(u()):.=\tilde{u}($. is nonanticipative. 
Proof. Consider the process $(x(),. u()$.$) on \left[t_{0}, T\right]$ such that $x\left(t_{0}\right)=x_{0}$. The construction of a feasible process $(\tilde{x}(),. \tilde{u}()$.$) can be provided following the lines of the proof of Lemma 3.2$ and Thm. 3.1, in which we do not fix an upper bound to the measure of the set in which we modify the reference control $u($.$) . More precisely, starting from (x(),. u()$.$) , we can construct a selection$ $\bar{u}($.$) as in case 2) of the proof of Lemma 3.2. Let M_{0}>0$ un upper bound for the velocities

of all trajectories having $x_{0}$ as left end-point. Take $\delta_{0} \in\left(0, \min \left\{\frac{\rho}{2 k_{h} M_{0}} ; \frac{\eta}{M_{0}^{2} k_{h}^{\prime}+k_{h} k_{f} M_{0}}\right\}\right)$ such that $\beta\left(\delta_{0}\right)<\eta$. Here, $k_{h}$ and $k_{h}^{\prime}$ the Lipschitz constants for $h($.$) and \nabla h($.$) respectively, on$ $e^{M T}\left(1+r_{0}\right) \mathbb{B}, \rho$ and $\eta$ are the positive constants provided by $(\mathrm{CQ})$, and $\beta($.$) is the positive$ function defined in (15) (cf. the proof of Lemma 3.2). It is not restrictive to assume that $-\rho / 2<h\left(x_{0}\right) \leq 0$. Then, we consider the process $(\tilde{x}(),. \tilde{u}()$.$) on \left[t_{0}, T\right]$ such that $\tilde{x}\left(t_{0}\right)=x_{0}$ and

$$
\tilde{u}(t):= \begin{cases}\bar{u}(t) & \text { if } t \in\left[t_{0}, T\right] \text { and } \frac{d}{d t} h(x(t))>-\eta \\ u(t) & \text { otherwise }\end{cases}
$$

where the control function $\bar{u}(.) \in \widetilde{U}($.$) is chosen to satisfy the inward pointing condition:$

$$
\nabla h(x(t)) \cdot \tilde{f}(t, x(t), \bar{u}(t)) \leq-\eta \quad \text { a.e. } t \in\left[t_{0}, T\right] .
$$

Observe that the construction of $\tilde{u}($.$) is nonanticipative and, for all t \in\left[t_{0},\left(t_{0}+\delta_{0}\right) \wedge T\right]$,

$$
d_{[\tau, t]}(u(.), \tilde{u}(.))=\operatorname{meas}\left\{\widetilde{\mathcal{A}}(t):=\left\{s \in\left[t_{0}, t\right]: \frac{d}{d t} h(x(t))>-\eta\right\}\right\} .
$$

Gronwall's inequality yields

$\|x(.)-\tilde{x}(.)\|_{L^{\infty}(\tau, t)} \leq 2 M\left(1+R_{0}\right) e^{\int_{0}^{T} \tilde{k}_{f}(s) d s} d_{[\tau, t]}(u(),. \tilde{u}())=.2 M\left(1+R_{0}\right) e^{\int_{0}^{T} \tilde{k}_{f}(s) d s} \operatorname{meas}\{\tilde{\mathcal{A}}(t)\}$.

The same analysis leading to inequalities (21) and (22) now gives:

$$
h(\tilde{x}(t))<0 \quad \text { for all } t \in\left(t_{0},\left(t_{0}+\delta_{0}\right) \wedge T\right] .
$$

The recursive argument appearing in the proof of Thm. 3.1 may be used, once again, to extend the process $(\tilde{x}(),. \tilde{u}()$.$) , preserving non-anticipativity. The proof of the proposition is complete.$

\subsection{Construction of nonanticipative maps and strategies}

Consider system (1), and fix an initial time $t_{0} \in[0, T]$ and a control $\tilde{v}(.) \in \mathcal{V}\left[t_{0}, T\right]$. Observe that assumptions (H1)-(H3) and (CQ1) guarantee that conditions (H1)'-(H3)' and (CQ) are satisfied by the function

$$
\tilde{f}(t, x, u):=f(x, u, \tilde{v}(t)) .
$$

The same property is valid when we fix a control $\tilde{u}(.) \in \mathcal{U}\left[t_{0}, T\right]$ and consider the function $(t, x, v) \rightarrow f(x, \tilde{u}(t), v)$. A consequence of Prop. 3.3 is the following proposition.

Proposition 3.4 Assume that (H1)-(H4) are satisfied. Take any $\left(t_{0}, x_{0}\right) \in[0, T] \times A$.

(i): If in addition (CQ1) is satisfied, then

$$
\mathcal{V}\left(t_{0}, x_{0}\right)=\mathcal{V}\left[t_{0}, T\right], \quad \mathcal{U}\left(\left[t_{0}, T\right], x_{0}\right) \neq \emptyset \quad \text { and } \quad S_{U}\left(t_{0}, x_{0}\right) \neq \emptyset
$$

In particular, there exists a nonanticipative strategy $\Gamma_{t_{0}, x_{0}} \in S_{U}\left(t_{0}, x_{0}\right)$ such that for any $v(.) \in \mathcal{V}\left(t_{0}, x_{0}\right)$ we have $x(t) \in \operatorname{int} A$ for all $t \in\left(t_{0}, T\right]$, where $x(t):=x\left[t_{0}, x_{0} ; \Gamma_{t_{0}, x_{0}}(v)(),. v().\right](t)$, for $t \in\left[t_{0}, T\right]$. 
(ii): If in addition (CQ2) is satisfied, then

$$
\mathcal{U}\left(t_{0}, x_{0}\right)=\mathcal{U}\left[t_{0}, T\right], \quad \mathcal{V}\left(\left[t_{0}, T\right], x_{0}\right) \neq \emptyset \quad \text { and } \quad S_{V}\left(t_{0}, x_{0}\right) \neq \emptyset
$$

In particular, there exists a nonanticipative strategy $\tilde{\Gamma}_{t_{0}, x_{0}} \in S_{V}\left(t_{0}, x_{0}\right)$ such that for any $u(.) \in \mathcal{U}\left(t_{0}, x_{0}\right)$ we have $x(t) \in \operatorname{int} A$ for all $t \in\left(t_{0}, T\right]$, where $x(t):=x\left[t_{0}, x_{0} ; u(),. \tilde{\Gamma}_{t_{0}, x_{0}}(u)().\right](t)$, for $t \in\left[t_{0}, T\right]$.

Proof. We show only (i), since (ii) can be proved in a similar way. Fix $\hat{u} \in \mathcal{U}\left[t_{0}, T\right]$. Take any $v(.) \in \mathcal{V}\left[t_{0}, T\right]$. Then we can consider

$$
\tilde{f}(t, x, u):=f(x, u, v(t)) .
$$

and apply Prop. 3.3 to obtain a feasible control $\gamma_{t_{0}, x_{0}}(\hat{u})(.) \in \mathcal{U}\left(\left[t_{0}, T\right], x_{0}\right)$. We define

$$
\Gamma_{t_{0}, x_{0}}(v)(.):=\gamma_{t_{0}, x_{0}}(\hat{u})(.) .
$$

It immediately follows that $\mathcal{V}\left(t_{0}, x_{0}\right)=\mathcal{V}\left[t_{0}, T\right], \mathcal{U}\left(\left[t_{0}, T\right], x_{0}\right) \neq \emptyset$ and $x(t) \in$ int $A$ for all $t \in\left(t_{0}, T\right]$, where $x():.=x\left[t_{0}, x_{0} ; \Gamma_{t_{0}, x_{0}}(v)(),. v().\right]($.$) . It remains to show that \Gamma_{t_{0}, x_{0}}$ is nonanticipative. Take $v_{1}(),. v_{2}(.) \in \mathcal{V}\left[t_{0}, T\right]$. If $v_{1}()=.v_{2}($.$) a.e. on \left[t_{0}, t_{0}+\tau\right]$, for some $\tau \in\left[0, T-t_{0}\right]$. Write $\tilde{f}_{1}(t, x, u):=f\left(x, u, v_{1}(t)\right)$ and $\tilde{f}_{2}(t, x, u):=f\left(x, u, v_{2}(t)\right)$. Let $\gamma_{t_{0}, x_{0}}^{1}$ and $\gamma_{t_{0}, x_{0}}^{2}$ be the nonanticipative maps provided by Prop. 3.3 and associated respectively with $\tilde{f}_{1}$ and $\tilde{f}_{2}$. Since $\tilde{f}_{1}(t, x, u)=\tilde{f}_{2}(t, x, u)$ a.e. on $\left[t_{0}, t_{0}+\tau\right]$, it immediately follows that

$$
\gamma_{t_{0}, x_{0}}^{1}(\hat{u})(t)=\gamma_{t_{0}, x_{0}}^{2}(\hat{u})(t), \quad \text { for a.e. } t \in\left[t_{0}, t_{0}+\tau\right]
$$

and therefore

$$
\Gamma_{t_{0}, x_{0}}\left(v_{1}\right)(t)=\Gamma_{t_{0}, x_{0}}\left(v_{2}\right)(t), \quad \text { for a.e. } t \in\left[t_{0}, t_{0}+\tau\right],
$$

confirming that $\Gamma_{t_{0}, x_{0}} \in S_{U}\left(t_{0}, x_{0}\right)$.

In Section 1 we have introduced the notion of nonanticipative strategies $S_{U}$ and $S_{V}$ for the two players controlling in system (1). We shall make use also of an extension to this concept, introducing a notion of nonanticipative maps between sets of admissible controls, not necessarily employed by different players, and having possibly different initial times. Take a pair of initial data points $\left(t_{1}, x_{1}\right),\left(t_{2}, x_{2}\right) \in[0, T] \times A$. If conditions (H1)-(H4) and (CQ1) are satisfied, from Prop. 3.4, we know that $\mathcal{U}\left(t_{1}, x_{1}\right) \neq \emptyset$ and $\mathcal{U}\left(t_{2}, x_{2}\right) \neq \emptyset$. A map $\gamma: \mathcal{U}\left(t_{1}, x_{1}\right) \rightarrow \mathcal{U}\left(t_{2}, x_{2}\right)$ is called nonanticipative if for any $\tau \in\left[0, T-t_{1}\right]$, given $u, u^{\prime} \in \mathcal{U}\left(t_{1}, x_{1}\right)$ such that $u()=.u^{\prime}($.$) a.e.$ on $\left[t_{1}, t_{1}+\tau\right]$, then also $\gamma(u)()=.\gamma\left(u^{\prime}\right)($.$) a.e. on \left[t_{2},\left(t_{2}+\tau\right) \wedge T\right]$. An equivalent notion is valid for maps $\gamma^{\prime}: \mathcal{V}\left(t_{1}, x_{1}\right) \rightarrow \mathcal{V}\left(t_{2}, x_{2}\right)$.

We draw attention to an immediate consequence of Thm. 3.1, Props. 3.3 and 3.4 summarized as the following corollary.

Corollary 3.5 Fix any $r_{0}>0$. Assume that (H1)-(H4) and (CQ1) are satisfied. Then there exists a constant $K>0$ such that for any initial time $t_{0} \in[0, T]$, for any $x_{1}, x_{2} \in A \cap r_{0} \mathbb{B}$, and 
for any $v(.) \in \mathcal{V}\left[t_{0}, T\right]$, we can find a nonanticipative map $\gamma_{v}: \mathcal{U}\left(t_{0}, x_{1}\right) \longrightarrow \mathcal{U}\left(t_{0}, x_{2}\right)$ with the following property: for any $u_{1}(.) \in \mathcal{U}\left(t_{0}, x_{1}\right)$ we have

$$
\begin{aligned}
& d_{\left[t_{0}, T\right]}\left(u_{1}(.), \gamma_{v}\left(u_{1}\right)(.)\right) \leq K\left|x_{1}-x_{2}\right|, \\
& \left\|x_{1}(.)-x_{2}(.)\right\|_{L^{\infty}\left(t_{0}, T\right)} \leq K\left|x_{1}-x_{2}\right| .
\end{aligned}
$$

where $x_{1}($.$) and x_{2}($.$) are the trajectories associated with the admissible controls \left(u_{1}(),. v().\right) \in$ $\mathcal{A D}\left(t_{0}, x_{1}\right)$ and $\left(u_{2}():.=\gamma_{v}\left(u_{1}\right)(),. v().\right) \in \mathcal{A D}\left(t_{0}, x_{2}\right)$ for system (1).

Remark 3.6 Cor. 3.5 has an obvious symmetric counterpart in which we can take any $x_{1}$, $x_{2} \in A \cap r_{0} \mathbb{B}$ and any $u(.) \in \mathcal{U}\left[t_{0}, T\right]$, obtaining a nonanticipative map $\gamma_{u}: \mathcal{V}\left(t_{0}, x_{1}\right) \longrightarrow \mathcal{V}\left(t_{0}, x_{2}\right)$ and estimates as (27) and (28).

\section{Properties of the lower and upper value functions}

Proposition 4.1 (Dynamic Programming Principle) Assume (H1)-(H4). For any $\left(t_{0}, x_{0}\right) \in$ $[0, T] \times A$ and for all $\sigma \in\left(0, T-t_{0}\right]$ we have the following properties:

(i): if in addition (CQ1) is satisfied, then

$$
\begin{gathered}
V^{\mathrm{b}}\left(t_{0}, x_{0}\right)=\inf _{\alpha \in S_{U}\left(\left[t_{0}, t_{0}+\sigma\right], x_{0}\right)} \sup _{v \in \mathcal{V}\left(t_{0}, x_{0}\right)}\left\{\int_{t_{0}}^{t_{0}+\sigma} L\left(t, x\left[t_{0}, x_{0} ; \alpha(v), v\right](t), \alpha(v)(t), v(t)\right) d t+\right. \\
\left.+V^{\mathrm{b}}\left(t_{0}+\sigma, x\left[t_{0}+\sigma, x_{0} ; \alpha(v), v\right]\left(t_{0}+\sigma\right)\right)\right\} .
\end{gathered}
$$

(ii): if in addition (CQ2) is satisfied, then

$$
\begin{gathered}
V^{\sharp}\left(t_{0}, x_{0}\right)=\sup _{\beta \in S_{V}\left(\left[t_{0}, t_{0}+\sigma\right], x_{0}\right)} \inf _{u \in \mathcal{U}\left(t_{0}, x_{0}\right)}\left\{\int_{t_{0}}^{t_{0}+\sigma} L\left(t, x\left[t_{0}, x_{0} ; u, \beta(u)\right](t), u(t), \beta(u)(t)\right) d t+\right. \\
\left.\quad+V^{\sharp}\left(t_{0}+\sigma, x\left[t_{0}+\sigma, x_{0} ; u, \beta(u)\right]\left(t_{0}+\sigma\right)\right)\right\},
\end{gathered}
$$

The proof of Prop. 4.1 is based on standard arguments and there is no substantial difference from the case without state constraints (cf. [2, 11, 14, 16, 19]).

Proposition 4.2 (Lipschitz continuity) Suppose that assumptions (H1)-(H4) and (CQ1) (respectively (CQ2)) are satisfied. Then, the lower value function $V^{b}$ (respectively the upper value function $\left.V^{\sharp}\right)$ is locally Lipschitz continuous on $[0, T] \times A$.

Proof. We prove the local Lipschitz regularity just for $V^{b}$, since the case for $V^{\sharp}$ can be treated in a similar way.

Fix any $r_{0}>0$. Let $\left(t_{0}, x_{0}\right),\left(t_{1}, x_{1}\right) \in[0, T] \times\left(A \cap r_{0} \mathbb{B}\right)$. Write $R_{0}:=e^{M T}\left(1+r_{0}\right), T_{1}:=$ $\left(T+t_{1}-t_{2}\right) \wedge T$ and $T_{2}:=\left(T+t_{2}-t_{1}\right) \wedge T$. For any given $\varepsilon>0$, invoking the definition of $V^{b}$, there exists a nonanticipative strategy $\alpha_{1} \in S_{U}\left(t_{1}, x_{1}\right)$, such that

$$
V^{b}\left(t_{1}, x_{1}\right)+\varepsilon \geq \sup _{v \in \mathcal{V}\left(t_{1}, x_{1}\right)} J\left(t_{1}, x_{1}, \alpha_{1}(v), v\right) .
$$


We claim that there exist a constant $K>0$, which depends only on the data of the problem, a nonanticipative map $\phi: \mathcal{V}\left(t_{2}, x_{2}\right) \rightarrow \mathcal{V}\left(t_{1}, x_{1}\right)$ and a nonanticipative strategy $\alpha_{2} \in S_{U}\left(t_{2}, x_{2}\right)$ such that, for all $v(.) \in \mathcal{V}\left(t_{2}, x_{2}\right)=\mathcal{V}\left[t_{2}, T\right]$, we have:

$$
d_{\left[t_{1}, T_{1}\right]}\left(\alpha_{1}(\phi(v))(.), \alpha_{2}(v)\left(.-t_{1}+t_{2}\right)\right) \leq K\left|x_{1}-x_{2}\right|,
$$

and

$$
\left\|x_{1}(.)-x_{2}\left(.-t_{1}+t_{2}\right)\right\|_{L^{\infty}\left(t_{1}, T_{1}\right)} \leq K\left|x_{1}-x_{2}\right|,
$$

in which $x_{1}(t):=x\left[t_{1}, x_{1} ; \alpha_{1}(\phi(v)), \phi(v)\right](t)$ and $x_{2}(t):=x\left[t_{2}, x_{2} ; \alpha_{2}(v), v\right](t)$.

We start defining the nonanticipative map $\phi: \mathcal{V}\left(t_{2}, x_{2}\right) \rightarrow \mathcal{V}\left(t_{1}, x_{1}\right)$. We observe that only one of the following two cases might occur: either $T_{1}<T$ or $T_{1}=T$.

If $T_{1}<T$ (i.e. $T_{2}=T$ ), then we fix a measurable function $\hat{v} \in \mathcal{V}\left[T_{1}, T\right]$ and, for all $v(.) \in$ $\mathcal{V}\left(t_{2}, x_{2}\right)=\mathcal{V}\left[t_{2}, T\right]$, we set

$$
\phi(v)(t):=\left\{\begin{array}{lll}
v\left(t-t_{1}+t_{2}\right) & \text { on } & {\left[t_{1}, T_{1}\right],} \\
\hat{v}(t) & \text { on } & \left(T_{1}, T\right],
\end{array}\right.
$$

On the other hand, if $T_{1}=T$, then, for all $v(.) \in \mathcal{V}\left(t_{2}, x_{2}\right)=\mathcal{V}\left[t_{2}, T\right]$, we write

$$
\phi(v)(.):=\left.v\left(.-t_{1}+t_{2}\right)\right|_{\left[t_{1}, T\right]} .
$$

Observe that the map $\mathcal{V}\left(t_{2}, x_{2}\right) \rightarrow \mathcal{A D}\left(t_{1}, x_{1}\right)$ defined by

$$
v \rightarrow\left(\alpha_{1}(\phi(v)), \phi(v)\right)
$$

is nonanticipative and can be modified giving a nonanticipative map $\mathcal{V}\left(t_{2}, x_{2}\right) \rightarrow \mathcal{A D}\left(t_{2}, x_{1}\right)$ as follows:

$$
v \rightarrow\left(\tilde{\alpha}_{1}(\phi(v)), v\right)
$$

in which, if $T_{1}<T$, then

$$
\tilde{\alpha}_{1}(\phi(v))(.):=\left.\alpha_{1}(\phi(v))\left(.-t_{2}+t_{1}\right)\right|_{\left[t_{2}, T\right]} \cdot
$$

Otherwise, if $T_{1}=T$ (i.e. $T_{2}<T$ ), then we set

$$
\tilde{\alpha}_{1}(\phi(v))(t)=\left\{\begin{array}{lll}
\alpha_{1}(\phi(v))\left(t-t_{2}+t_{1}\right) & \text { on } & {\left[t_{2}, T_{2}\right],} \\
\Gamma_{T_{2}, x_{2}\left(T_{2}\right)}(v)(t) & \text { on } & \left(T_{2}, T\right],
\end{array}\right.
$$

in which $x_{2}\left(T_{2}\right):=x\left[t_{2}, x_{2} ; \alpha_{1}(\phi(v)), v\right]\left(T_{2}\right)$ and $\Gamma_{T_{2}, x_{2}\left(T_{2}\right)}$ is the nonanticipative map defined in (26) (for a fixed measurable function $\hat{u} \in \mathcal{U}\left[T_{2}, T\right]$ ).

Given any $v \in \mathcal{V}\left(t_{2}, x_{2}\right)$ we consider the nonanticipative map $\gamma_{v}: \mathcal{U}\left(t_{2}, x_{2}\right) \rightarrow \mathcal{U}\left(t_{2}, x_{1}\right)$ provided by Cor. 3.5. It follows that the map $\mathcal{V}\left(t_{2}, x_{2}\right) \rightarrow \mathcal{A D}\left(t_{2}, x_{2}\right)$ defined by

$$
v \rightarrow\left(\gamma_{v}\left(\tilde{\alpha}_{1}(\phi(v))\right), v\right)
$$

is non anticipative and, setting $\alpha_{2}(v):=\gamma_{v}\left(\tilde{\alpha}_{1}(\phi(v))\right)$ we obtain a strategy satisfying all the requirements of the claim, and, in particular, estimates (31) and (32).

Using again the definition of $V^{b}$, employing the strategy $\alpha_{2} \in S_{U}\left(t_{2}, x_{2}\right)$ constructed above, we have

$$
V^{b}\left(t_{2}, x_{2}\right) \leq \sup _{v \in \mathcal{V}\left(t_{2}, x_{2}\right)} J\left(t_{2}, x_{2}, \alpha_{2}(v), v\right)
$$


And, therefore, there exists a feasible control $\bar{v}_{2} \in \mathcal{V}\left(t_{2}, x_{2}\right)=\mathcal{V}\left[t_{2}, T\right]$ such that

$$
V^{b}\left(t_{2}, x_{2}\right) \leq J\left(t_{2}, x_{2}, u_{2}, \alpha_{2}\left(\bar{v}_{2}\right), \bar{v}_{2}\right)+\varepsilon \text {. }
$$

Write $\bar{x}_{1}(t):=x\left[t_{1}, x_{1} ; \alpha_{1}\left(\phi\left(\bar{v}_{2}\right)\right), \phi\left(\bar{v}_{2}\right)\right](t)$ and $\bar{x}_{2}(t):=x\left[t_{2}, x_{2} ; \alpha_{2}\left(\bar{v}_{2}\right), \bar{v}_{2}\right](t)$. Owing to the inequalities above, bearing in mind estimates (31)-(32), the Lipschitz continuity properties of $L$ and $g$ (see condition (H3)) and the upper bound $M\left(1+R_{0}\right)$ for both the velocity of trajectories emanating from $A \cap r_{0} \mathbb{B}$ and for the Lagrangian $L$ along these trajectories (see (H2)), some routine analysis yields

$$
\begin{aligned}
& V^{b}\left(t_{2}, x_{2}\right)-V^{b}\left(t_{1}, x_{1}\right) \leq J\left(t_{2}, x_{2}, \alpha_{2}\left(\bar{v}_{2}\right), \bar{v}_{2}\right)-J\left(t_{1}, x_{1}, \alpha_{1}\left(\phi\left(\bar{v}_{2}\right)\right), \phi\left(\bar{v}_{2}\right)\right)+2 \varepsilon \\
& \leq \int_{t_{2}}^{T} L\left(t, \bar{x}_{2}(t), \alpha_{2}\left(\bar{v}_{2}\right)(t), \bar{v}_{2}(t)\right) d t \\
& -\int_{t_{1}}^{T} L\left(t, \bar{x}_{1}(t), \alpha_{1}\left(\phi\left(\bar{v}_{2}\right)\right)(t), \phi\left(\bar{v}_{2}\right)(t)\right) d t+g\left(\bar{x}_{1}(T)\right)-g\left(\bar{x}_{2}(T)\right)+2 \varepsilon \\
& \leq \int_{t_{1}}^{T_{1}} \mid L\left(t, \bar{x}_{1}(t), \alpha_{1}\left(\phi\left(\bar{v}_{2}\right)\right)(t), \phi\left(\bar{v}_{2}\right)(t)\right) \\
& -L\left(t+t_{2}-t_{1}, \bar{x}_{2}\left(t+t_{2}-t_{1}\right), \alpha_{2}\left(\bar{v}_{2}\right)\left(t+t_{2}-t_{1}\right), \bar{v}_{2}\left(t+t_{2}-t_{1}\right)\right) \mid d t \\
& +M\left(1+R_{0}\right)\left|t_{1}-t_{2}\right|+k_{g} K\left|x_{1}-x_{2}\right|+k_{g} M\left(1+R_{0}\right)\left|t_{1}-t_{2}\right|+2 \varepsilon \\
& \leq \int_{t_{1}}^{T_{1}}\left|L\left(t, \bar{x}_{1}(t), \alpha_{1}\left(\phi\left(\bar{v}_{2}\right)\right)(t), \phi\left(\bar{v}_{2}\right)(t)\right)-L\left(t, \bar{x}_{1}(t), \alpha_{2}\left(\bar{v}_{2}\right)\left(t+t_{2}-t_{1}\right), \phi\left(\bar{v}_{2}\right)(t)\right)\right| d t \\
& +\int_{t_{1}}^{T_{1}} \mid L\left(t, \bar{x}_{1}(t), \alpha_{2}\left(\bar{v}_{2}\right)\left(t+t_{2}-t_{1}\right), \phi\left(\bar{v}_{2}\right)(t)\right) \\
& -L\left(t+t_{2}-t_{1}, \bar{x}_{2}\left(t+t_{2}-t_{1}\right), \alpha_{2}\left(\bar{v}_{2}\right)\left(t+t_{2}-t_{1}\right), \bar{v}_{2}\left(t+t_{2}-t_{1}\right)\right) \mid d t \\
& +k_{g} K\left|x_{1}-x_{2}\right|+M\left(1+R_{0}\right)\left(1+k_{g}\right)\left|t_{1}-t_{2}\right|+2 \varepsilon \\
& \leq \int_{t_{1}}^{T_{1}} k_{L}\left(\left|t_{1}-t_{2}\right|+\left\|\bar{x}_{1}(.)-\bar{x}_{2}\left(.-t_{1}+t_{2}\right)\right\|_{L^{\infty}\left(t_{1}, T_{1}\right)}\right) d t+k_{g} K\left|x_{1}-x_{2}\right| \\
& +M\left(1+R_{0}\right) d_{\left[t_{1}, T_{1}\right]}\left(\alpha_{1}\left(\phi\left(\bar{v}_{2}\right)\right)(.), \alpha_{2}\left(\bar{v}_{2}\right)\left(.-t_{1}+t_{2}\right)\right)+M\left(1+R_{0}\right)\left(1+k_{g}\right)\left|t_{1}-t_{2}\right|+2 \varepsilon \\
& \leq T k_{L}\left(\left|t_{1}-t_{2}\right|+K\left|x_{1}-x_{2}\right|\right)+k_{g} K\left|x_{1}-x_{2}\right|+M\left(1+R_{0}\right)\left[\left(1+k_{g}\right)\left|t_{1}-t_{2}\right|\right. \\
& \left.+K\left|x_{1}-x_{2}\right|\right]+2 \varepsilon \text {. }
\end{aligned}
$$

Exchanging the roles of $\left(t_{1}, x_{1}\right)$ and $\left(t_{2}, x_{2}\right)$ in the above inequalities, and letting $\varepsilon \downarrow 0$ we finally obtain that

$$
\left|V^{\mathrm{b}}\left(t_{1}, x_{1}\right)-V^{\mathrm{b}}\left(t_{2}, x_{2}\right)\right| \leq K^{\mathrm{b}}\left(\left|t_{1}-t_{2}\right|+\left|x_{1}-x_{2}\right|\right),
$$

for some constant $K^{b}>0$ (which depends only on $r_{0}$ and the data of the differential game), confirming the proposition statement.

\subsection{Solutions of Hamilton-Jacobi-Isaacs equations}

Theorem 4.3 Assume that conditions (H1)-(H5) are satisfied. 
(i) If in addition (CQ1) is satisfied, then the lower value function $V^{b}$ is a viscosity supersolution on $[0, T) \times A$ and a viscosity subsolution on $[0, T) \times$ int $A$ of equation (6) in which $H=H^{b}$;

(ii) If in addition (CQ2) is satisfied, then the upper value function $V^{\sharp}$ is a viscosity supersolution on $[0, T) \times$ int $A$ and a viscosity subsolution on $[0, T) \times A$ of equation (6) in which $H=H^{\sharp}$.

Proof. We start observing that the continuity of $H^{b}$ and $H^{\sharp}$ follows immediately from Berge Maximum Theorem (cf. [1, Theorem 1.4.16]). We shall prove below part (i). Part (ii) is proved by applying part (i) to characterize $-V^{\sharp}(t, x)$ as the lower value function of a modified game, in which the original cost $J(., ., .,$.$) is replaced by -J(., ., .,$.$) , and using the fact that a subgradient$ of $-V^{\sharp}$ is expressible as $-\left(\xi_{0}, \xi_{1}\right)$ in which $\left(\xi_{0}, \xi_{1}\right)$ is a supergradient of $V^{\sharp}$ and vice-versa. Here, sub- and supergradients are understood in the sense of gradients of minorizing or majorizing $\mathcal{C}^{1}$ test functions, as in Def. 2.1.

1. $V^{b}$ is a viscosity supersolution on $[0, T) \times A$ of equation (6) with $H=H^{b}$.

Take any $\left(t_{0}, x_{0}\right) \in[0, T) \times A$ and let $\varphi: \mathbb{R} \times \mathbb{R}^{n} \rightarrow \mathbb{R}$ be a $\mathcal{C}^{1}$ function such that $V^{b}-\varphi$ has a local minimum at $\left(t_{0}, x_{0}\right)$ (relative to $[0, T] \times A$ ). It is not restrictive to assume that $V^{b}\left(t_{0}, x_{0}\right)=\varphi\left(t_{0}, x_{0}\right)$ and so there exists $r_{0} \in(0,1)$ such that $V^{b}(t, x) \geq \varphi(t, x)$, for all $(t, x) \in$ $\left(\left(t_{0}, x_{0}\right)+r_{0} \mathbb{B}\right) \cap([0, T] \times A)$. Suppose in contradiction that, for some $\theta>0$, we have

$$
H^{b}\left(t_{0}, x_{0}, \partial_{x} \varphi\left(t_{0}, x_{0}\right)\right)-\partial_{t} \varphi\left(t_{0}, x_{0}\right) \leq-\theta
$$

and so

$$
\inf _{v \in V} \sup _{u \in U} \mathcal{H}\left(t_{0}, x_{0}, \partial_{x} \varphi\left(t_{0}, x_{0}\right), u, v\right)-\partial_{t} \varphi\left(t_{0}, x_{0}\right) \leq-\theta .
$$

Take $v_{0} \in V$ such that

$$
\sup _{u \in U} \mathcal{H}\left(t_{0}, x_{0}, \partial_{x} \varphi\left(t_{0}, x_{0}\right), u, v_{0}\right)-\partial_{t} \varphi\left(t_{0}, x_{0}\right) \leq-\theta,
$$

and, by continuity of the functions involved in the expression above, we obtain, following a reduction in the size of $r_{0}>0$ if required,

$$
\sup _{u \in U} \mathcal{H}\left(t, x, \partial_{x} \varphi(t, x), u, v_{0}\right)-\partial_{t} \varphi(t, x) \leq-\frac{\theta}{2},
$$

for all $(t, x) \in\left(\left(t_{0}, x_{0}\right)+r_{0} \mathbb{B}\right) \cap([0, T] \times A)$. Define the control $\tilde{v}(.) \equiv v_{0}$. From Proposition 3.4 we know that the control $\tilde{v} \in \mathcal{V}(t, x)$. Since an upper bound can be established for the speed of all trajectories emanating from $\left(\left(t_{0}, x_{0}\right)+\mathbb{B}\right) \cap([0, T] \times A)$, from (36) we deduce that there exists $\sigma_{0} \in\left(0, T-t_{0}\right)$ such that for every strategy $\alpha \in S_{U}\left(t_{0}, x_{0}\right)$ and for every $s \in\left[0, \sigma_{0}\right]$, we have

$$
\mathcal{H}\left(s, x(s), \partial_{x} \varphi(s, x(s)), \alpha(\tilde{v})(s), \tilde{v}(s)\right)-\partial_{t} \varphi(s, x(s)) \leq-\frac{\theta}{2},
$$

where $x(s):=x\left[t_{0}, x_{0} ; \alpha(\tilde{v}), \tilde{v}\right](s)$. It follows that, for every strategy $\alpha \in S_{U}\left(t_{0}, x_{0}\right)$ and for every $\sigma \in\left[0, \sigma_{0}\right]$, we obtain

$$
\int_{t_{0}}^{t_{0}+\sigma}\left[\mathcal{H}\left(s, \tilde{x}(s), \partial_{x} \varphi(s, \tilde{x}(s)), \alpha(\tilde{v})(s), \tilde{v}(s)\right)-\partial_{t} \varphi(s, \tilde{x}(s))\right] d s \leq-\frac{\theta \sigma}{2},
$$

where $\tilde{x}(s):=x\left[t_{0}, x_{0} ; \alpha(\tilde{v}), \tilde{v}\right](s)$. 
On the other hand, invoking the Dynamic Programming Principle (Proposition 4.1), writing $x(s):=x\left[t_{0}, x_{0} ; \alpha(v), v\right](s)$, we deduce:

$$
\begin{aligned}
0= & \inf _{\alpha \in S_{U}\left(\left[t_{0}, t_{0}+\sigma\right], x_{0}\right)} \sup _{v \in \mathcal{V}\left(t_{0}, x_{0}\right)}\left\{\int_{t_{0}}^{t_{0}+\sigma} L(s, x(s), \alpha(v)(s), v(s)) d s+\right. \\
& \left.+V^{b}\left(t_{0}+\sigma, x\left(t_{0}+\sigma\right)\right)-V^{b}\left(t_{0}, x_{0}\right)\right\} \\
\geq & \inf _{\alpha \in S_{U}\left(\left[t_{0}, t_{0}+\sigma\right], x_{0}\right)} \sup _{v \in \mathcal{V}\left(t_{0}, x_{0}\right)}\left\{\int_{t_{0}}^{t_{0}+\sigma} L(s, x(s), \alpha(v)(s), v(s)) d s+\right. \\
& \left.+\varphi\left(t_{0}+\sigma, x\left(t_{0}+\sigma\right)\right)-\varphi\left(t_{0}, x_{0}\right)\right\} \\
= & \inf _{\alpha \in S_{U}\left(\left[t_{0}, t_{0}+\sigma\right], x_{0}\right)} \sup _{v \in \mathcal{V}\left(t_{0}, x_{0}\right)}\left\{\int_{t_{0}}^{t_{0}+\sigma}[L(s, x(s), \alpha(v)(s), v(s))\right. \\
& \left.\left.+\partial_{x} \varphi(s, x(s)) \cdot f(s, x(s), \alpha(v)(s), v(s))+\partial_{t} \varphi(s, x(s))\right] d s\right\} \\
= & \inf _{\alpha \in S_{U}\left(\left[t_{0}, t_{0}+\sigma\right], x_{0}\right)} \sup _{v \in \mathcal{V}\left(t_{0}, x_{0}\right)}\left\{-\int_{t_{0}}^{t_{0}+\sigma}\left[\mathcal{H}\left(s, x(s), \partial_{x} \varphi(s, x(s)), \alpha(v)(s), v(s)\right)\right.\right. \\
& \left.\left.-\partial_{t} \varphi(s, x(s))\right] d s\right\} \\
\geq & \inf _{\alpha \in S_{U}\left(\left[t_{0}, t_{0}+\sigma\right], x_{0}\right)}\left\{-\int_{t}^{t+\sigma}\left[\mathcal{H}\left(s, \tilde{x}(s), \partial_{x} \varphi(s, \tilde{x}(s)), \alpha(\tilde{v})(s), \tilde{v}(s)\right)-\partial_{t} \varphi(s, \tilde{x}(s))\right] d s\right\} .
\end{aligned}
$$

This relation together with (37) provides a contradiction.

2. $V^{b}$ is a viscosity subsolution on $[0, T) \times \operatorname{int} A$ of equation (6) with $H=H^{b}$.

Take any $\left(t_{0}, x_{0}\right) \in[0, T) \times \operatorname{int} A$ and let $\varphi: \mathbb{R} \times \mathbb{R}^{n} \rightarrow \mathbb{R}$ be a $\mathcal{C}^{1}$ function such that $V^{b}-\varphi$ has a local maximum at $\left(t_{0}, x_{0}\right)$ (relative to $\left.[0, T] \times A\right)$. Again it is not restrictive to assume that $V^{b}\left(t_{0}, x_{0}\right)=\varphi\left(t_{0}, x_{0}\right)$ and so there exists $r_{0} \in(0,1)$ such that $\left(\left(t_{0}, x_{0}\right)+r_{0} \mathbb{B}\right) \cap([0, T] \times A) \subset$ $[0, T] \times$ int $A$, and $V^{\mathrm{b}}(t, x) \leq \varphi(t, x)$ for all $(t, x) \in\left(\left(t_{0}, x_{0}\right)+r_{0} \mathbb{B}\right) \cap([0, T] \times A)$. Suppose in contradiction that, for some $\theta>0$, we have

$$
H^{b}\left(t_{0}, x_{0}, \partial_{x} \varphi\left(t_{0}, x_{0}\right)\right)-\partial_{t} \varphi\left(t_{0}, x_{0}\right) \geq \theta
$$

that is

$$
\inf _{v \in V} \sup _{u \in U} \mathcal{H}\left(t_{0}, x_{0}, \partial_{x} \varphi\left(t_{0}, x_{0}\right), u, v\right)-\partial_{t} \varphi\left(t_{0}, x_{0}\right) \geq \theta .
$$

We also have that the multifunction

$$
v \leadsto G(v):=\arg \max \left\{\mathcal{H}\left(t_{0}, x_{0}, \partial_{x} \varphi\left(t_{0}, x_{0}\right), u, v\right)-\partial_{t} \varphi\left(t_{0}, x_{0}\right) \mid u \in U\right\}
$$

takes values (non-empty) compact subsets of $U$ and is upper-semi-continuous (owing to Berge's Theorem). Then, classical results on set-valued maps (cf. [1, Prop. 8.2.1]) imply that $G($.$) is$ Borel-measurable and, therefore, there exists a Borel-measurable selection $\bar{u}(v) \in G(v)$ (cf. [1, Thm. 8.1.3] for a measurable selection theorem). Consider any control $v \in \mathcal{V}\left(t_{0}, x_{0}\right)$. The map (defined on $\left[t_{0}, T\right]$ )

$$
t \rightarrow \bar{u}(v(t)) \text { is measurable on }\left[t_{0}, T\right]
$$

and takes values in $U$. Observe also, from the construction above, that

$$
\mathcal{H}\left(t_{0}, x_{0}, \partial_{x} \varphi\left(t_{0}, x_{0}\right), \bar{u}(v(t)), v(t)\right)-\partial_{t} \varphi\left(t_{0}, x_{0}\right) \geq \theta .
$$


Write $\bar{x}():.=x\left[t_{0}, x_{0} ; \bar{u}(v), v\right]($.$) . From the regularity of \varphi$ and of the functions involved in the definition of $\mathcal{H}$, and since there exists an upper bound for the speed of all trajectories starting from $x_{0}$, we can find $\sigma_{0} \in\left(0,\left(T-t_{0}\right) \wedge r_{0} / 2\right)$ such that

$$
\bar{x}(s) \in x_{0}+\frac{r_{0}}{2} \mathbb{B} \subset \text { int } A, \quad \text { for all } s \in\left[t_{0}, t_{0}+\sigma_{0}\right],
$$

and

$$
\mathcal{H}\left(s, \bar{x}(s), \partial \varphi_{x}(s, \bar{x}(s)), \bar{u}(v(s)), v(s)\right)-\partial \varphi_{t}(s, \bar{x}(s)) \geq \frac{\theta}{2}, \quad \text { for all } s \in\left[t_{0}, t_{0}+\sigma_{0}\right] .
$$

Define now the map $\bar{\alpha}: \mathcal{V}\left(t_{0}, x_{0}\right) \rightarrow \mathcal{U}\left(t_{0}, x_{0}\right)$ as follows

$$
\bar{\alpha}(v)(t):=\left\{\begin{array}{l}
\bar{u}(v(t)) \quad \forall t \in\left[t_{0}, t_{0}+\sigma_{0}\right) \\
\Gamma_{t_{0}+\sigma_{0}, \bar{x}\left(t_{0}+\sigma_{0}\right)}(v)(t) \quad \forall t \in\left[t_{0}+\sigma_{0}, T\right],
\end{array}\right.
$$

where $\Gamma_{t_{0}+\sigma_{0}, x} \in S_{U}\left(t_{0}+\sigma_{0}, x\right)$ is the strategy defined as in (26) (we can always fix a measurable function $\hat{u} \in \mathcal{U}\left[t_{0}+\sigma_{0}, T\right]$ to construct $\Gamma_{t_{0}+\sigma_{0}, x}$, for all $x \in A$ ). Clearly $\bar{\alpha} \in S_{U}\left(t_{0}, x_{0}\right)$, and also $\bar{\alpha} \in S_{U}\left(\left[t_{0}, t_{0}+\sigma\right], x_{0}\right)$ for all $\sigma \in\left[0, \sigma_{0}\right]$. Now, for a given control $v \in \mathcal{V}\left(t_{0}, x_{0}\right)$, we still use notation $\bar{x}():.=x\left[t_{0}, x_{0} ; \bar{\alpha}(v), v\right]($.$) for the (admissible) trajectory which now involves the$ strategy-control pair $(\bar{\alpha}, v)$. From $(39)$ we immediately deduce that

$$
\mathcal{H}\left(s, \bar{x}(s), \partial \varphi_{x}(s, \bar{x}(s)), \bar{\alpha}(v)(s), v(s)\right)-\partial \varphi_{t}(s, \bar{x}(s)) \geq \frac{\theta}{2}, \quad \text { for all } s \in\left[t_{0}, t_{0}+\sigma_{0}\right] .
$$

Therefore, for all $\sigma \in\left[0, \sigma_{0}\right]$, integrating on $\left[t_{0}, t_{0}+\sigma\right]$, we obtain that

$$
\inf _{v \in \mathcal{V}\left(t_{0}, x_{0}\right)} \int_{t_{0}}^{t_{0}+\sigma}\left[\mathcal{H}\left(s, x(s), \partial_{x} \varphi(s, \bar{x}(s)), \bar{\alpha}(v)(s), v(s)\right)-\partial_{t} \varphi(s, \bar{x}(s))\right] d s \geq \frac{\theta \sigma}{2}
$$

But, the Dynamic Programming Principle Prop. 4.1 (writing $x(s):=x\left[t_{0}, x_{0} ; \alpha(v), v\right](s)$ ) yields:

$$
\begin{aligned}
0= & \inf _{\alpha \in S_{U}\left(\left[t_{0}, t_{0}+\sigma\right], x_{0}\right)} \sup _{v \in \mathcal{V}\left(t_{0}, x_{0}\right)}\left\{\int_{t_{0}}^{t_{0}+\sigma} L(s, x(s), \alpha(v)(s), v(s)) d s+\right. \\
& \left.+V^{\mathrm{b}}\left(t_{0}+\sigma, x\left(t_{0}+\sigma\right)\right)-V^{b}\left(t_{0}, x_{0}\right)\right\} \\
\leq & \inf _{\alpha \in S_{U}\left(\left[t_{0}, t_{0}+\sigma\right], x_{0}\right)} \sup _{v \in \mathcal{V}\left(t_{0}, x_{0}\right)}\left\{\int_{t_{0}}^{t_{0}+\sigma} L(s, x(s), \alpha(v)(s), v(s)) d s+\right. \\
& \left.+\varphi\left(t_{0}+\sigma, x\left(t_{0}+\sigma\right)\right)-\varphi\left(t_{0}, x_{0}\right)\right\} \\
= & \inf _{\alpha \in S_{U}\left(\left[t_{0}, t_{0}+\sigma\right], x_{0}\right)} \sup _{v \in \mathcal{V}\left(t_{0}, x_{0}\right)}\left\{-\int_{t_{0}}^{t_{0}+\sigma}\left[\mathcal{H}\left(s, x(s), \partial_{x} \varphi(s, x(s)), \alpha(v)(s), v(s)\right)\right.\right. \\
& \left.\left.-\partial_{t} \varphi(s, x(s))\right] d s\right\} \\
\leq & \sup _{v \in \mathcal{V}\left(t_{0}, x_{0}\right)}\left\{-\int_{t}^{t+\sigma}\left[\mathcal{H}\left(s, \bar{x}(s), \partial_{x} \varphi(s, \bar{x}(s)), \bar{\alpha}(v)(s), v(s)\right)-\partial_{t} \varphi(s, \bar{x}(s))\right] d s\right\} .
\end{aligned}
$$


We have arrived at a contradiction to (40) and the proof is complete.

Proposition 4.4 Assume that conditions (H1)-(H5) are satisfied.

(i) If in addition (CQ1) and (CQ3) are satisfied, then the lower value function $V^{b}$ is simultaneously a viscosity supersolution and a viscosity subsolution on $[0, T) \times A$ of equation (6) in which $H=H^{b}$;

(ii) If in addition (CQ2) and (CQ4) are satisfied, then the upper value function $V^{\sharp}$ is simultaneously a viscosity supersolution and a viscosity subsolution on $[0, T) \times A$ of equation (6) in which $H=H^{\sharp}$.

Proof. Again we provide just the proof of (i) since (ii) can be derived arguing in a similar way. The fact that $V^{b}$ is a viscosity supersolution on $[0, T) \times A$ of equation (6) with $H=H^{b}$ has been already established in Thm. 4.3. So we have to prove that $V^{b}$ is a viscosity subsolution on $[0, T) \times A$ of equation (6) with $H=H^{b}$.

Take any $\left(t_{0}, x_{0}\right) \in[0, T) \times A$ and let $\varphi: \mathbb{R} \times \mathbb{R}^{n} \rightarrow \mathbb{R}$ be a $\mathcal{C}^{1}$ function such that $V^{\mathrm{b}}-\varphi$ has a local maximum at $\left(t_{0}, x_{0}\right)$ (relative to $\left.[0, T] \times A\right)$. We can restrict attention to the case when $x_{0} \in \partial A$, since otherwise, if $x_{0} \in$ int $A$, then the analysis is like in step $\mathbf{2}$ of the proof of Thm. 4.3. And it is not restrictive to assume that $V^{b}\left(t_{0}, x_{0}\right)=\varphi\left(t_{0}, x_{0}\right)$ and that there exists $r_{0} \in(0,1)$ such that $V^{b}(t, x) \leq \varphi(t, x)$ for all $(t, x) \in\left(\left(t_{0}, x_{0}\right)+r_{0} \mathbb{B}\right) \cap([0, T] \times A)$. Suppose in contradiction that, for some $\theta>0$, we have

$$
\theta \leq H^{b}\left(t_{0}, x_{0}, \partial_{x} \varphi\left(t_{0}, x_{0}\right)\right)-\partial_{t} \varphi\left(t_{0}, x_{0}\right)=\inf _{v \in V} \sup _{u \in U} \mathcal{H}\left(t_{0}, x_{0}, \partial_{x} \varphi\left(t_{0}, x_{0}\right), u, v\right)-\partial_{t} \varphi\left(t_{0}, x_{0}\right) .
$$

Invoking (CQ3) we can find $u_{0} \in U, v_{0} \in V$ and $\eta_{0}>0$ such that, eventually reducing the size of the radius $r_{0}$, from the continuity of $\mathcal{H}, \partial_{t} \varphi$ and $\partial_{x} \varphi$, we obtain

$$
\mathcal{H}\left(t, x, \partial_{x} \varphi(t, x), u_{0}, v_{0}\right)-\partial_{t} \varphi(t, x) \geq \frac{\theta}{2},
$$

and

$$
\nabla h(x) \cdot f\left(x, u_{0}, v_{0}\right)<-\eta_{0},
$$

for all $(t, x) \in\left(\left(t_{0}, x_{0}\right)+r_{0} \mathbb{B}\right) \cap([0, T] \times A)$. We also know that we can find $\sigma_{0} \in\left(0,\left(T-t_{0}\right) \wedge r_{0} / 2\right)$ such that all trajectories $x(.) \mathrm{s}$ starting from $x_{0}$ satisfy the inclusion $x(s) \in x_{0}+\frac{r_{0}}{2} \mathbb{B}$ for all $s \in\left[t_{0}, t_{0}+\sigma_{0}\right]$. Fix the control $\tilde{u}(.) \equiv u_{0}$ and consider the admissible strategy for player one $\alpha_{0} \in S_{U}\left(t_{0}, x_{0}\right)$ defined as in (26) of the proof of Prop. 3.4 taking $\tilde{u}(.) \equiv u_{0}$ as reference control:

$$
\alpha_{0}(.):=\Gamma_{t_{0}, x_{0}}(v)(.)=\gamma_{t_{0}, x_{0}}\left(\tilde{u} \equiv u_{0}\right)(.) \text {. }
$$

Observe that $\alpha_{0} \in S_{U}\left(t_{0}+\sigma_{0}, x_{0}\right)$ and, from (42) and the construction of $\gamma_{t_{0}, x_{0}}\left(\tilde{u} \equiv u_{0}\right)$, for the (constant) control $\tilde{v}(.) \equiv v_{0} \in \mathcal{V}\left(t_{0}, x_{0}\right)$ we obtain $\alpha_{0}\left(\tilde{v} \equiv v_{0}\right)(s)=u_{0}$ for a.e. $s \in\left[t_{0}, t_{0}+\sigma_{0}\right]$. Therefore, invoking once again the Dynamic Programming Principle Prop. 4.1 and using inequality (41) (writing $x():.=x\left[t_{0}, x_{0} ; \alpha(v), v\right](),. \tilde{x}():.=x\left[t_{0}, x_{0} ; \alpha_{0}(v), v\right]($.$) and \tilde{x}_{0}():.=$ $x\left[t_{0}, x_{0} ; \alpha_{0}\left(\tilde{v} \equiv v_{0}\right), \tilde{v} \equiv v_{0}\right]($.$) to make the notation simpler), for all \sigma \in\left[0, \sigma_{0}\right]$ we arrive at the 
following sequence of inequalities:

$$
\begin{aligned}
0 \leq & \inf _{\alpha \in S_{U}\left(\left[t_{0}, t_{0}+\sigma\right], x_{0}\right)} \sup _{v \in \mathcal{V}\left(t_{0}, x_{0}\right)}\left\{-\int_{t_{0}}^{t_{0}+\sigma}\left[\mathcal{H}\left(s, x(s), \partial_{x} \varphi(s, x(s)), \alpha(v)(s), v(s)\right)\right.\right. \\
& \left.\left.-\partial_{t} \varphi(s, x(s))\right] d s\right\} \\
\leq & \sup _{v \in \mathcal{V}\left(t_{0}, x_{0}\right)}\left\{-\int_{t}^{t+\sigma}\left[\mathcal{H}\left(s, \tilde{x}(s), \partial_{x} \varphi(s, \tilde{x}(s)), \alpha_{0}(v)(s), v(s)\right)-\partial_{t} \varphi(s, \tilde{x}(s))\right] d s\right\} \\
\leq & \left\{-\int_{t}^{t+\sigma}\left[\mathcal{H}\left(s, \tilde{x}_{0}(s), \partial_{x} \varphi\left(s, \tilde{x}_{0}(s)\right), \alpha_{0}\left(v_{0}\right)(s), v_{0}\right)-\partial_{t} \varphi\left(s, \tilde{x}_{0}(s)\right)\right] d s\right\} \\
\leq & -\frac{\theta}{2} \sigma<0 .
\end{aligned}
$$

This is a contradiction and the proof is complete.

Immediate consequences of Thm. 4.3 and Prop. 4.4 are the following corollaries.

Corollary 4.5 Assume that (H1)-(H5), (CQ1) and (CQ2) hold true. Suppose moreover that the Isaacs condition

$$
H^{b}(t, x, p)=H^{\sharp}(t, x, p), \quad \forall(t, x, p) \in[0, T) \times A \times \mathbb{R}^{n}
$$

is satisfied. Then,

(i) the lower value function $V^{b}$ is a viscosity supersolution on $[0, T) \times A$ and a viscosity subsolution on $[0, T) \times \operatorname{int} A$ of equation (6) also with $H=H^{\sharp}$

(ii) the upper value function $V^{\sharp}$ is a viscosity supersolution on $[0, T) \times$ int $A$ and a viscosity subsolution on $[0, T) \times A$ of equation (6) also with $H=H^{b}$.

Corollary 4.6 Assume that (H1)-(H5) and (CQ1)-(CQ2) hold true. Suppose moreover that the Isaacs condition

$$
H^{b}(t, x, p)=H^{\sharp}(t, x, p), \quad \forall(t, x, p) \in[0, T) \times A \times \mathbb{R}^{n}
$$

and (CQ3) (or equivalently (CQ4)) are satisfied. Then,

(i) the lower value function $V^{b}$ is a viscosity solution on $[0, T) \times A$ of equation (6) also with $H=H^{\sharp}$

(ii) the upper value function $V^{\sharp}$ is a viscosity solution on $[0, T) \times A$ of equation (6) also with $H=H^{b}$.

\section{Comparison and uniqueness results}

Theorem 5.1 Assume that conditions (H1)-(H5) are satisfied and that $A$ is compact.

(i) Suppose in addition that (CQ1) is satisfied and consider equation (6) in which we take $H=H^{b}$. Take two continuous functions $W_{1}, W_{2}:[0, T] \times A \longrightarrow \mathbb{R}$ satisfying the following properties

(a) $W_{1}(T,)=.W_{2}(T,).(=g()$.$) on A$; 
(b) $W_{1}(t, x)$ is a viscosity subsolution on $[0, T) \times$ int $A$ of equation (6);

(c) $W_{2}(t, x)$ is a viscosity supersolution on $[0, T) \times A$ of equation (6).

Then we obtain:

$$
W_{1}(t, x) \leq W_{2}(t, x), \quad \forall(t, x) \in[0, T] \times A .
$$

(ii) Suppose in addition that (CQ2) is satisfied and consider equation (6) in which we take $H=H^{\sharp}$. Take two continuous functions $W_{1}, W_{2}:[0, T] \times A \longrightarrow \mathbb{R}$ satisfying the properties (a)-(c) of (i) above. Then we obtain:

$$
W_{1}(t, x) \leq W_{2}(t, x), \quad \forall(t, x) \in[0, T] \times A .
$$

Proof. We shall prove only part (i) of Thm. 5.1, in which we take the Hamiltonian to be $H=H^{b}$. The proof of part (ii) is similar.

1. Suppose, in contradiction, that $\sup _{(t, x) \in[0, T] \times A} W_{1}(t, x)-W_{2}(t, x)>0$. Then, there would exist a point $\left(\bar{s}_{0}, \bar{x}_{0}\right) \in[0, T) \times A$ such that:

$$
W_{1}\left(\bar{s}_{0}, \bar{x}_{0}\right)-W_{2}\left(\bar{s}_{0}, \bar{x}_{0}\right)>0 .
$$

We now follow an approach based on a Kruzkov type transform. Let $M \in \mathbb{R}$ be a lower bound for both $W_{1}$ and $W_{2}$. We can choose the constant $M$ in such a manner that $M \leq 0$ and

$$
L(s, x, u, v)-M \geq 1, \quad \text { for all } u \in U, v \in V,
$$

for all $s \in[0, T]$, and $x \in A$. Take a positive constant $c$ such that $c \geq 1-M T$. Define the functions

$$
\widetilde{W}_{i}(t, x):=\frac{1}{1+t} \log \left(W_{i}(T-t, x)+M(T-t)-M+c\right), \quad i=1,2,
$$

and the Hamiltonian $\widetilde{H}^{b}: \mathbb{R}^{1+n+1+1+n} \rightarrow \mathbb{R}:$

$$
\widetilde{H}^{b}\left(t, x, w, p_{t}, p_{x}\right):=\inf _{v \in V} \sup _{u \in U} \widetilde{\mathcal{H}}\left(t, x, w, p_{t}, p_{x}, u, v\right),
$$

where

$$
\widetilde{\mathcal{H}}\left(t, x, w, p_{t}, p_{x}, u, v\right):=(1+t) p_{t}-(1+t) f(x, u, v) \cdot p_{x}-\frac{L(T-t, x, u, v)-M}{e^{(1+t) w}} .
$$

Write $\bar{t}_{0}:=T-\bar{s}_{0}(>0)$. Observe that, passing to functions $\widetilde{W}_{i}$ 's (which are continuous on $[0, T] \times A)$, from (43) and the compactness of $A$ there would exist a number $\sigma>0$ and a point $(\bar{t}, \bar{x}) \in(0, T] \times A$ such that:

$$
\sigma:=\widetilde{W}_{1}(\bar{t}, \bar{x})-\widetilde{W}_{2}(\bar{t}, \bar{x})=\max _{(t, x) \in[0, T] \times A}\left(\widetilde{W}_{1}(t, x)-\widetilde{W}_{2}(t, x)\right) \geq \widetilde{W}_{1}\left(\bar{t}_{0}, \bar{x}_{0}\right)-\widetilde{W}_{2}\left(\bar{t}_{0}, \bar{x}_{0}\right)>0 .
$$

We restrict attention to the case when ' $\bar{x} \in \partial A$ ', since dealing with the case ' $\bar{x} \in \operatorname{int} A$ ' is similar, but simpler (cf. [2], [14]).

We claim that $\widetilde{W}_{1}$ and $\widetilde{W}_{2}$ satisfy the conditions for being a viscosity subsolution on $(0, T) \times \operatorname{int} A$, and a viscosity supersolution on $(0, T] \times A$, respectively, of

$$
\left\{\begin{aligned}
W(t, x)+\widetilde{H}^{b}\left(t, x, W(t, x), \partial_{t} W(t, x), \partial_{x} W(t, x)\right) & =0 & \text { on }(0, T) \times A \\
W(0, x) & =\tilde{g}(x) & \text { on } A,
\end{aligned}\right.
$$


where $\tilde{g}(x):=\log (g(x)-M+c)$.

Indeed, assume that $\left(t_{0}, x_{0}\right) \in(0, T] \times A$ is a local minimizer for $\widetilde{W}_{2}-\psi$ where $\psi$ is a $\mathcal{C}^{1}$ function. It is not restrictive to suppose that $\left(\widetilde{W}_{2}-\psi\right)\left(t_{0}, x_{0}\right)=0$ and, so, locally we have $\widetilde{W}_{2} \geq \psi$. Consider the $\mathcal{C}^{1}$ test function $\varphi(s, x):=e^{(1+T-s) \psi(T-s, x)}-M s+M-c$. From the monotonicity of the exponential function, we obtain that $\left(T-t_{0}, x_{0}\right)$ is a local minimizer for $(t, x) \rightarrow\left(W_{2}-\varphi\right)(t, x)$. Consequently, since $W_{2}$ is a viscosity supersolution of $(6)$, we have

$$
H^{b}\left(T-t_{0}, x_{0}, \partial_{x} \varphi\left(T-t_{0}, x_{0}\right)\right)-\partial_{t} \varphi\left(T-t_{0}, x_{0}\right) \geq 0
$$

which yields

$$
\inf _{v \in V} \sup _{u \in U} \mathcal{H}\left(T-t_{0}, x_{0}, \partial_{x} \varphi\left(T-t_{0}, x_{0}\right), u, v\right)-\partial_{s} \varphi\left(T-t_{0}, x_{0}\right) \geq 0 .
$$

On the other hand, from the definition of $\varphi$, we also have:

$$
\begin{aligned}
& \partial_{s} \varphi\left(T-t_{0}, x_{0}\right)=-e^{\left(1+t_{0}\right) \psi\left(t_{0}, x_{0}\right)}\left[\left(1+t_{0}\right) \partial_{t} \psi\left(t_{0}, x_{0}\right)+\psi\left(t_{0}, x_{0}\right)\right]-M ; \\
& \partial_{x} \varphi\left(T-t_{0}, x_{0}\right)=e^{\left(1+t_{0}\right) \psi\left(t_{0}, x_{0}\right)}\left(1+t_{0}\right) \partial_{x} \psi\left(t_{0}, x_{0}\right) .
\end{aligned}
$$

Substituting these quantities in (47), we obtain

$$
\begin{aligned}
0 \leq & \psi\left(t_{0}, x_{0}\right)+\inf _{v \in V} \sup _{u \in U}\left\{\left(1+t_{0}\right) \partial_{t} \psi\left(t_{0}, x_{0}\right)\right. \\
& \left.\quad-\left(1+t_{0}\right) f\left(x_{0}, u, v\right) \cdot \partial_{x} \psi\left(t_{0}, x_{0}\right)-\frac{L\left(T-t_{0}, x_{0}, u, v\right)-M}{e^{\left(1+t_{0}\right) \psi\left(t_{0}, x_{0}\right)}}\right\} \\
= & \psi\left(t_{0}, x_{0}\right)+\widetilde{H}^{b}\left(t_{0}, x_{0}, \psi\left(t_{0}, x_{0}\right), \partial_{t} \psi\left(t_{0}, x_{0}\right), \partial_{x} \psi\left(t_{0}, x_{0}\right)\right) .
\end{aligned}
$$

which yields that $\widetilde{W}_{2}$ is a viscosity supersolution of $(46)$ on $(0, T] \times A$.

The fact that $\widetilde{W}_{1}$ is a viscosity subsolution on $(0, T) \times$ int $A$ of (46) can be shown by similar techniques. So we omit its proof.

2. This step consists in selecting suitable test functions. Define $\bar{\xi}:=-\nabla h(\bar{x})\left(\in \operatorname{int} T_{A}(\bar{x})\right)$. From the characterization of the interior of the Clarke tangent cone of $A$ at $\bar{x}, T_{A}(\bar{x})$, (cf. [20]), we can find constants $\delta \in(0,1)$ and $\eta \in(0,1)$ such that

$$
z+(0, \delta](\bar{\xi}+\eta \mathbb{B}) \subset \operatorname{int} A, \quad \text { for all } z \in(\bar{x}+2 \delta \mathbb{B}) \cap A .
$$

Write $\omega():. \mathbb{R}_{+} \rightarrow \mathbb{R}_{+}$for a modulus of continuity for functions $\widetilde{W}_{i}$ 's. Notice that $\omega($.$) can be$ considered bounded from above by a constant $C>0$, since the $\widetilde{W}_{i}$ 's are bounded on $[0, T] \times A$.

For any $n \in \mathbb{N}$ fixed, we define the continuous (bounded from above) function $\phi_{n}$ depending on the variables $s, t \in[0, T], x, y \in A$ :

$$
\phi_{n}(s, t, x, y):=\widetilde{W}_{1}(s, x)-\widetilde{W}_{2}(t, y)-n^{2}\left|x-y-\frac{1}{n} \bar{\xi}\right|^{2}-n^{2}\left|s-t+\frac{1}{\sqrt{n}}\right|^{2}-|y-\bar{x}|^{2}-|t-\bar{t}|^{2} .
$$

Let $\left(s_{n}, t_{n}, x_{n}, y_{n}\right) \in[0, T]^{2} \times A^{2}$ be a point of maximum for $\phi_{n}$, which exists since $A$ is compact:

$$
\phi_{n}\left(s_{n}, t_{n}, x_{n}, y_{n}\right)=\max _{(s, t, x, y) \in[0, T]^{2} \times A^{2}} \phi_{n}(s, t, x, y) .
$$


Observe that, for all $n>\max \left\{\frac{1}{\delta} ;\left(\frac{4}{\bar{t}}\right)^{2}\right\},\left(\bar{t}-\frac{1}{\sqrt{n}}, \bar{x}+\frac{1}{n} \bar{\xi}\right) \in(0, T) \times \operatorname{int} A$ and we have

$$
\phi_{n}\left(s_{n}, t_{n}, x_{n}, y_{n}\right) \geq \phi_{n}\left(\bar{t}-\frac{1}{\sqrt{n}}, \bar{t}, \bar{x}+\frac{1}{n} \bar{\xi}, \bar{x}\right) .
$$

We claim that

$$
\lim _{n \rightarrow \infty} x_{n}=\lim _{n \rightarrow \infty} y_{n}=\bar{x} \quad \text { and } \quad \lim _{n \rightarrow \infty} s_{n}=\lim _{n \rightarrow \infty} t_{n}=\bar{t} .
$$

Indeed, from (48) and (49), for each $n>\max \left\{\frac{1}{\delta} ;\left(\frac{4}{t}\right)^{2}\right\}$ we obtain

$$
\begin{aligned}
0 \leq & \left.\widetilde{W}_{1}\left(s_{n}, x_{n}\right)-\widetilde{W}_{2}\left(t_{n}, y_{n}\right)\right)-\left[\widetilde{W}_{1}\left(\bar{t}-\frac{1}{\sqrt{n}}, \bar{x}+\frac{1}{n} \bar{\xi}\right)-\widetilde{W}_{2}(\bar{t}, \bar{x})\right] \\
& -n^{2}\left|x_{n}-y_{n}-\frac{1}{n} \bar{\xi}\right|^{2}-n^{2}\left|s_{n}-t_{n}+\frac{1}{\sqrt{n}}\right|^{2}-\left|y_{n}-\bar{x}\right|^{2}-\left|t_{n}-\bar{t}\right|^{2} .
\end{aligned}
$$

As a consequence, since from $(45)$ the term $\left(\widetilde{W}_{1}\left(s_{n}, x_{n}\right)-\widetilde{W}_{2}\left(s_{n}, x_{n}\right)\right)-\left(\widetilde{W}_{1}(\bar{t}, \bar{x})-\widetilde{W}_{2}(\bar{t}, \bar{x})\right) \leq 0$, and making use of the modulus of continuity $\omega($.$) (which is bounded by C$ ), we deduce that

$$
\begin{aligned}
n^{2} \mid x_{n} & -y_{n}-\left.\frac{1}{n} \bar{\xi}\right|^{2}+n^{2}\left|s_{n}-t_{n}+\frac{1}{\sqrt{n}}\right|^{2}+\left|y_{n}-\bar{x}\right|^{2}+\left|t_{n}-\bar{t}\right|^{2} \\
& \leq \omega\left(\left|\left(s_{n}-t_{n}, x_{n}-y_{n}\right)\right|\right)+\omega\left(\left|\left(\frac{1}{\sqrt{n}}, \frac{\bar{\xi}}{n}\right)\right|\right) \\
& \leq 2 C .
\end{aligned}
$$

It follows that

$$
\begin{aligned}
& \left|x_{n}-y_{n}-\frac{1}{n} \bar{\xi}\right|,\left|s_{n}-t_{n}+\frac{1}{\sqrt{n}}\right| \leq \frac{\sqrt{2 C}}{n} \\
& \left|y_{n}-\bar{x}\right|,\left|t_{n}-\bar{t}\right| \leq \sqrt{2 C} \\
& \left|x_{n}-y_{n}\right| \leq \frac{1}{n}(\sqrt{2 C}+|\bar{\xi}|) \\
& \left|s_{n}-t_{n}\right| \leq \frac{1}{\sqrt{n}}\left(\frac{\sqrt{2 C}}{\sqrt{n}}+1\right) .
\end{aligned}
$$

Extracting a subsequence, we obtain in the limit

$$
\lim _{n \rightarrow \infty} x_{n}=\lim _{n \rightarrow \infty} y_{n}=\tilde{x} \quad \text { and } \lim _{n \rightarrow \infty} s_{n}=\lim _{n \rightarrow \infty} t_{n}=\tilde{t},
$$

for some $(\tilde{t}, \tilde{x}) \in[0, T] \times A$. Using again (50), and observing that

$$
\lim _{n \rightarrow \infty}\left[\omega\left(\left|\left(s_{n}-t_{n}, x_{n}-y_{n}\right)\right|\right)+\omega\left(\left|\left(\frac{1}{\sqrt{n}}, \frac{\bar{\xi}}{n}\right)\right|\right)\right]=0,
$$

we deduce that $(\tilde{t}, \tilde{x})=(\bar{t}, \bar{x})$.

3. Take $\bar{n} \in \mathbb{N}$ large enough such that

$$
\bar{n}>\max \left\{\frac{1}{\delta} ;\left(\frac{4}{\bar{t}}\right)^{2}\right\}
$$


and, for all $n \geq \bar{n}$, we have:

$$
\omega\left(\left|\left(s_{n}-t_{n}, x_{n}-y_{n}\right)\right|\right)+\omega\left(\left|\left(\frac{1}{\sqrt{n}}, \frac{\bar{\xi}}{n}\right)\right|\right) \leq \min \left\{\eta^{2} ; \delta^{2} ; \frac{\bar{t}^{2}}{4}\right\} .
$$

Considering (50) with this choice of $\bar{n}$, we see that, for all $n \geq \bar{n}$,

$$
\begin{gathered}
0<t_{n}, \quad 0<s_{n}<T, \quad y_{n} \in(\bar{x}+\delta \mathbb{B}) \cap A, \\
x_{n} \in y_{n}+\frac{1}{n}[\bar{\xi}+\eta \mathbb{B}] \subset y_{n}+\delta[\bar{\xi}+\eta \mathbb{B}] \subset \operatorname{int} A .
\end{gathered}
$$

As a consequence $\left(s_{n}, x_{n}\right) \in(0, T) \times \operatorname{int} A$, and $\left(t_{n}, y_{n}\right) \in(0, T] \times A$.

Fix $n \in \mathbb{N}$ with $n \geq \bar{n}$. We define the test functions $\psi_{1}, \psi_{2}:[0, T] \times \mathbb{R}^{n} \rightarrow \mathbb{R}$ as follows:

$$
\psi_{1}(s, x):=\widetilde{W}_{2}\left(t_{n}, y_{n}\right)+n^{2}\left|x-y_{n}-\frac{1}{n} \bar{\xi}\right|^{2}+n^{2}\left|s-t_{n}+\frac{1}{\sqrt{n}}\right|^{2}+\left|y_{n}-\bar{x}\right|^{2}+\left|t_{n}-\bar{t}\right|^{2},
$$

and

$$
\psi_{2}(t, y):=\widetilde{W}_{1}\left(s_{n}, x_{n}\right)-n^{2}\left|x_{n}-y-\frac{1}{n} \bar{\xi}\right|^{2}-n^{2}\left|s_{n}-t+\frac{1}{\sqrt{n}}\right|^{2}+|y-\bar{x}|^{2}+|t-\bar{t}|^{2} .
$$

Observe that the point $\left(s_{n}, x_{n}\right)$ is a local maximizer for the function $\widetilde{W}_{1}-\psi_{1}$ (on $\left.[0, T] \times A\right)$. Since $\widetilde{W}_{1}$ is a viscosity subsolution of $(46)$ on $(0, T) \times \operatorname{int} A$, it immediately follows that

$$
\widetilde{W}_{1}\left(s_{n}, x_{n}\right)+\widetilde{H}^{b}\left(s_{n}, x_{n}, \widetilde{W}_{1}\left(s_{n}, x_{n}\right), \partial_{s} \psi_{1}\left(s_{n}, x_{n}\right), \partial_{x} \psi_{1}\left(s_{n}, x_{n}\right)\right) \leq 0 .
$$

Similarly, since $\left(t_{n}, y_{n}\right)$ is a local minimizer for the function $\widetilde{W}_{2}-\psi_{2}$ on $[0, T] \times A$, and $\widetilde{W}_{2}$ is a viscosity supersolution of $(46)$ on $(0, T] \times A$, we also have

$$
0 \leq \widetilde{W}_{2}\left(t_{n}, y_{n}\right)+\widetilde{H}^{b}\left(t_{n}, y_{n}, \widetilde{W}_{2}\left(t_{n}, y_{n}\right), \partial_{t} \psi_{2}\left(t_{n}, y_{n}\right), \partial_{y} \psi_{2}\left(t_{n}, y_{n}\right)\right)
$$

Taking into account inequalities (53) and (54), for all $n \geq \bar{n}$ we obtain:

$$
\begin{aligned}
\widetilde{W}_{1}\left(s_{n}, x_{n}\right)-\widetilde{W}_{2}\left(t_{n}, y_{n}\right) \leq & \widetilde{H}^{b}\left(t_{n}, y_{n}, \widetilde{W}_{2}\left(t_{n}, y_{n}\right), \partial_{t} \psi_{2}\left(t_{n}, y_{n}\right), \partial_{y} \psi_{2}\left(t_{n}, y_{n}\right)\right) \\
& -\widetilde{H}^{b}\left(s_{n}, x_{n}, \widetilde{W}_{1}\left(s_{n}, x_{n}\right), \partial_{s} \psi_{1}\left(s_{n}, x_{n}\right), \partial_{x} \psi_{1}\left(s_{n}, x_{n}\right)\right) .
\end{aligned}
$$

Letting $n \rightarrow+\infty$, taking into account the regularity properties of all the functions involved $\left(\widetilde{W}_{i}\right.$ and $\psi_{i}$ for $i=1,2$ and $\widetilde{H}^{b}$ ), it follows that

$$
\begin{aligned}
0 & <\sigma=\widetilde{W}_{1}(\bar{t}, \bar{x})-\widetilde{W}_{2}(\bar{t}, \bar{x}) \\
& \leq \widetilde{H}^{b}\left(\bar{t}, \bar{x}, \widetilde{W}_{2}(\bar{t}, \bar{x}), \partial_{t} \psi_{2}(\bar{t}, \bar{x}), \partial_{x} \psi_{2}(\bar{t}, \bar{x})\right)-\widetilde{H}^{b}\left(\bar{t}, \bar{x}, \widetilde{W}_{1}(\bar{t}, \bar{x}), \partial_{t} \psi_{1}(\bar{t}, \bar{x}), \partial_{x} \psi_{1}(\bar{t}, \bar{x})\right)
\end{aligned}
$$

Therefore, considering the expressions for the derivatives of $\psi_{1}$ and $\psi_{2}$, bearing in mind the limit behaviour of the sequences $\left(s_{n}, x_{n}\right)$ and $\left(t_{n}, y_{n}\right)$ (cf. also (50) and (51)), using the definition of the Hamiltonian $\widetilde{H}^{b}$, the inequality (55) above, for suitable $\bar{u} \in U$ and $\bar{v} \in V$, implies

$$
\begin{aligned}
0<\sigma & \leq-\frac{L(T-\bar{t}, \bar{x}, \bar{u}, \bar{v})-M}{e^{(1+\bar{t}) \widetilde{W}_{2}(\bar{t}, \bar{x})}}-\frac{L(T-\bar{t}, \bar{x}, \bar{u}, \bar{v})-M}{e^{(1+\bar{t}) \widetilde{W}_{1}(\bar{t}, \bar{x})}} \\
& =(L(T-\bar{t}, \bar{x}, \bar{u}, \bar{v})-M) \frac{e^{\widetilde{W}_{2}(\bar{t}, \bar{x})}-e^{\widetilde{W}_{1}(\bar{t}, \bar{x})}}{e^{\widetilde{W}_{1}(\bar{t}, \bar{x})+\widetilde{W}_{2}(\bar{t}, \bar{x})}} .
\end{aligned}
$$


From (44) it follows that

$$
e^{\widetilde{W}_{2}(\bar{t}, \bar{x})}-e^{\widetilde{W}_{1}(\bar{t}, \bar{x})}>0
$$

But this contradicts (45).

We conclude that $W_{1}(t, x)-W_{2}(t, x) \leq 0$ for all $(t, x) \in[0, T] \times A$, confirming the assertions of the theorem.

Observe that in step 2 of the proof of Thm. 5.1 we can replace the continuous function $\phi_{n}$ defined in (48) by the function $\tilde{\phi}_{n}$ (still depending on the variables $s, t \in[0, T], x, y \in A$ ), in which we have exchanged $x$ and $y$ in the term $n^{2}\left|y-x-\frac{1}{n} \bar{\xi}\right|^{2}$ :

$$
\tilde{\phi}_{n}(s, t, x, y):=\widetilde{W}_{1}(s, x)-\widetilde{W}_{2}(t, y)-n^{2}\left|y-x-\frac{1}{n} \bar{\xi}\right|^{2}-n^{2}\left|s-t+\frac{1}{\sqrt{n}}\right|^{2}-|y-\bar{x}|^{2}-|t-\bar{t}|^{2} .
$$

It is then easy to check that this minor modification produces a sequence $\left\{\left(s_{n}, t_{n}, x_{n}, y_{n}\right)\right\}$ in $[0, T]^{2} \times A^{2}$ such that, this time, we have $y_{n} \in \operatorname{int} A$ for each $n$ large enough. This suits very well with the fact that $\left(t_{n}, y_{n}\right)$ is a local minimizer for the function $\widetilde{W}_{2}-\tilde{\psi}_{2}$ (where $\tilde{\psi}_{2}$ is a function obtained by a suitable modification of $\psi_{2}$ ), and now $\widetilde{W}_{2}$ is a viscosity supersolution of (46) (on $(0, T] \times \operatorname{int} A)$. Then, arguing as in the proof of Thm. 5.1, we obtain the following proposition.

Proposition 5.2 Assume that conditions (H1)-(H5) are satisfied and that $A$ is compact. Then all the assertions of Thm. 5.1 remain valid when we replace (b) and (c) by (b)' and (c)' below:

$(b)^{\prime} W_{1}(t, x)$ is a viscosity subsolution on $[0, T) \times A$ of equation (6);

$(c)^{\prime} W_{2}(t, x)$ is a viscosity supersolution on $[0, T) \times$ int $A$ of equation (6).

Acknowledgments. This research of the first and second authors benefited from the support of the "FMJH Program Gaspard Monge in optimization and operation research" (PGMO 2015-2832H, PGMO 2016-1570H), and from the support to this program from EDF and EDFTHALES-ORANGE-CRITEO. The research of the second author benefited from the support of the project PGMO 2018-0047H, and was also partially supported by research contract AFOSRFA9550-18-1-0254.

\section{References}

[1] J.-P. Aubin and H. Frankowska, Set-valued Analysis, Birkhäuser Boston, Inc., Boston, Basel, Berlin, 1990.

[2] M. Bardi and I. Capuzzo-Dolcetta, Optimal control and viscosity solutions of the Hamilton-Jacobi equations., Birkhäuser, Boston, 1997.

[3] M. Bardi, S. Koike and P. Soravia, Pursuit-evasion game with state constraints: dynamic programming and discrete-time approximations, Discrete Contin. Dynam. Systems, vol. 6, no. 2, 2000, pp. 361-380.

[4] T. Basar, P. Bernhard, H-infinity Optimal Control and Related Minimax Design Problems Birkhaser, Boston, Massachusetts, 1991.

[5] P. Bettiol, P. Cardaliaguet and M. Quincampoix, Zero-sum state constrained differential games: existence of value for Bolza problem, Int. J. Game Theory, vol. 34, 2006, pp. 495-527. 
[6] P. Bettiol, P. Cardaliaguet and M. Quincampoix, On a Non anticipative Filippov Theorem for Control Systems with state constraints, Proceedings of the 44th IEEE Conference on Decision and Control, and the European Control Conference, CDCECC '05, 2006, pp. 257-261.

[7] P. Bettiol and H. Frankowska, Regularity of solution maps of differential inclusions for systems under state constraints, Journal of Set-Valued Analysis, vol. 15, no. 1, 2007, pp. 21-45.

[8] P. Bettiol and R. B. Vinter, Trajectories Satisfying a Smooth State Constraint: Improved Estimates, IEEE TAC, vol. 56, no. 5, 2011, pp. 1090-1096.

[9] P. Bettiol and R. B. Vinter, Estimates on trajectories in a closed set with corners for $(t, x)$ dependent data, Mathematical Control and Related Fields, vol. 3, 2013, pp. $245-267$.

[10] P. Cardaliaguet, M. Quincampoix and P. Saint-Pierre, Numerical methods for differential games, in "Stochastic and differential games: Theory and numerical methods", Annals of the international Society of Dynamic Games, M. Bardi, T.E.S. Raghavan, T. Parthasarathy Eds. Birkhäuser, 1999, pp. 177-247.

[11] P. Cardaliaguet, M. Quincampoix and P. Saint-Pierre, Pursuit differential games with state constraints., SIAM J. Control Optim., vol. 39, no. 5, 2001, pp. 1615-1632.

[12] P. Cardaliaguet and S. Plaskacz, Invariant solutions of differential games and Hamilton-Jacobi equations for time-measurable hamiltonians, SIAM J. Control Optim., vol.38, no. 5, 2000, pp. 1501-1520

[13] C. Edwards, S. Spurgeon, Sliding Mode Control: Theory And Applications, CRC Press, Boca Raton, Florida, 1998.

[14] L. C. Evans and P. E. Souganidis, Differential games and representation formulas for solutions of Hamilton-Jacobi Equations, Indiana Univ. Math. J., vol. 282, 1984, pp. 487-502.

[15] R. Isaacs, Differential Games, Wiley, New York, 1965.

[16] S. Koike, On the state constraint problem for differential games. Indiana Univ. Math. J., vol. 44, no. 2, 1995, pp. 467-487.

[17] N. N. Krasovskii and A. I. Subbotin, Game-Theorical Control Problems, SpringerVerlag, New-York, 1988.

[18] H. Frankowska and M. Mazzola, On relations of the adjoint state to the value function for optimal control problems with state constraints, NoDEA Nonlinear Differential Equations Appl., vol. 20, no. 2, 2013, pp. 361-383.

[19] S. Plaskacz and M. Quincampoix, Value-functions for differential games and control systems with discontinuous terminal cost, SIAM J. Control Optim., vol. 39, no. 5, 2000, pp. 1485-1498.

[20] R. T. Rockafellar Clarke's tangent cones and the boundaries of closed sets in $\mathbb{R}^{n}$, Nonlinear Anal., vol. 3, no. 1, 1979, pp. 145-154.

[21] H. M. Soner Optimal control problems with state-space constraints, SIAM J. Control Optim., vol. 24, 1986, pp. 552-562. 
[22] R. B. Vinter, Optimal Control, Birkhaüser, Boston, 2000. 